\title{
Semiclassical theory of shot noise in mesoscopic conductors
}

\author{
M.J.M. de Jong ${ }^{a} \mathrm{~b} *$, C.W.J. Beenakker ${ }^{\mathrm{b}}$ \\ 'Philps Rescanch Laboratones 5656 AA Lindhoven The Netherlands \\ ${ }^{\mathrm{b}}$ Instituut-Lorentz Universitj of Laden 2300 RA Leiden The Nctherlands
}

Recerved 29 January 1996

\begin{abstract}
A scmiclassical theory is developed for trme-dependent current fluctuations in mesoscopic conductors The theory is based on the Boltzmann Langevin equation for a degenerate election gas The low-fiequency shot-noise powet is iclated to classical tiansmission probabilities at the Feimi level For a disordered conductor with impuity scattering, it is shown how the shot norse ciosses over from zelo in the ballistic legime to one-thild of the Poisson noise in the diffusive 1egime In a conductor consisting of $n$ tunnel bailess in seires, the shot noise approaches one-thutd of the Poisson norse as $n$ gocs to infintty, independent of the transpatency of the batters The analysis confirms that phase coherence is not required for the occurience of the one-third suppression of the shot noise The effects of clection heating and inclastic scatteing are calculated, by inseiting charge-conserving election icseivolis between segments of the conductor
\end{abstract}

PACS $7350 \mathrm{Td}, 7210 \mathrm{Bg}, 7270+\mathrm{m}, 7323 \mathrm{Ps}$

$K e$ ulords Noise and fluctuations, Electionic tianspoit theory

\section{Introduction}

The discieteness of the election chatge causes time-dependent fluctuations in the clectrical curient, known as shot noise These fluctuations ate chaldeterized by a white norse spectium and peisist down to zero temperature The shot-norse power $P$ contarns information on the conduction piocess, which is not given by the resistance $\Lambda$ woll-known example is a vacuum diode, whele $P=2 e|\bar{I}| \equiv P_{\text {Polsson }}$, with $\bar{I}$ the average curient This tells us that the elections traverse the conductor in a completely

\footnotetext{
${ }^{*}$ Coltesponding duthor Tel $+31402472069, \mathrm{fax}+31402473365$

c mal jongmim (a) natlab iescarch philips com
} 
uncorrelated fashion, as in a Poisson process In macroscopic samples, the shot noise is averaged out to zero by inelastic scatteing

In the past few years, the shot norse has been investigated in mesoscopic conductors, smaller than the inelastic scattering length Theoretical analysis shows that the

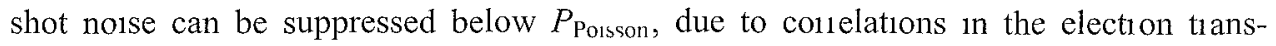
mission imposed by the Paulı pinciple [1-5] Most intiiguingly, it has been found that $P=\frac{1}{3} P_{\text {Poisson }}$ in a metallic, diffusive conductor [6-10] The factor one-thind is univeisal in the sense that it is independent of the material, sample size, or degiee of disorder, as long as the length $L$ of the conductor is greater than the mean free path $f$ and shoiter than the localization length An observation of suppressed shot noise in a diffusive conductor has been repotted [11] In a quantum mechanical descisption [6], the suppiession follows fiom the bimodal distıbution of tiansmission eigenvalues [12] Surprisingly, Nagaev [7] finds the same onc-thind suppiession fiom a scmiclassical approach, in which the Pauli punciple is accounted for, but the motion of elections is treated classically This implies that phase coherence is not essential for the suppression A similar conclusion is obtained in Ref [13] However, the relationship between the quantum mechanical and semiclassical theor ies remains unclear [14]

In this paper, we 1emvestigate the semiclassical appioach and present a deta1ed compaison with quantum mechanical calculations in the literatuie In paiticulat, we study how the shot noise crosses over from the ballistic to the diffusive regime This complements the study of the crossover of the conductance in Ref [15] We use the Boltzmann-Langevin equation $[16,17]$, which is a semiclassical kinetic equation for nonequilibium fiuctuations This equation has pieviously been applied to shot noise by Kul1k and Omel'yanchuk [18] for a ballistic point contact, and by Nagaev [7] for a diffusive conductor Here, we will demonstrate how the Boltzmann-Langevin equation can be applied to an arbitiaiy mesoscopic conductor Ou analysis conects pievious work by Beenakke1 and Van Houten [19] A biref account of our main results has been repoited in Ref [20]

The outline of this paper is as follows In Section 2 we discuss the BoltzmannLangevin equation It is demonstiated how the shot-norse power can be expicssed in terms of semiclassical transmission probabilities Impuity scattering is ticated in Section 3 The shot noise power incicases fiom zelo in the ballistic legime to $\frac{1}{3} P_{\text {Porson }}$ 1n the diffusive regime We consider both isotiopic and nonisotiopic impuity scatteing, and both a two- and three-dimensional density of states We also piesent a one-dimensional model, which can be solved analytically Exact agiecment is found with a previous quantum mechanical evaluation [8], in the limit of a conductance $\gg e^{2} / h$ Section 4 deals with bairie1 scatteing We consider tunneling thiough $n$ planat batileis in selies (tunnel probability $\Gamma$ ) For $n=2$ and $\Gamma \ll 1$, we recovel the resulıs for a double-bai1er junction of Refs [21,22] In the limit $n \rightarrow \infty$ the shotnorse power appioaches $\frac{1}{3} P_{\text {Porson }}$ independent of $\Gamma$ By taking the continuum limit, $n \rightarrow \infty, \Gamma \rightarrow 1$, at fixed $n(1-\Gamma)$, we tecover the onc-dimensional model of Section 3 The case of a disordered iegion in selies with a tunnel bailiei concludes Section 4 In Section 5 we calculate the effects of melastic scattening and of election hedting 
due to electron electron scatteing Analogous to the work of Beenakker and Buttıker [6], this scattering is modeled by putting charge-conserving electron 1eservours between phase-coherent segments of the conductor This allows us to model the effects of quasielastic scatteung, election heatıng, and inelastic scatteing withın a single theoretical framework We conclude in Section 6

Before pioceeding with a desciption of the semiclassical appioach, we birefly summan ize the fully quantum mechanıcal theory The zero-temperature, zero-fiequency shotnoise power $P$ of a phase-coherent conductor is related to the transmission matix $\mathrm{t}$ by the formula [4]

$$
P=P_{0} \operatorname{Tr} \mathrm{t}^{\dagger}\left(1-\mathrm{tt}^{\dagger}\right)=P_{0} \sum_{n-1}^{N} T_{n}\left(1-T_{n}\right),
$$

where $P_{0} \equiv 2 e|V| G_{0}$, with $V$ the applied voltage and $G_{0} \equiv e^{2} / h$ the conductance quantum (we assume spinless electrons for simplicity of notation), $T_{n} \in[0,1]$ an eigenvalue of $\mathrm{t} \mathrm{t}^{\dagger}$, and $N$ the number of transverse modes at the Ferm energy $E_{\mathrm{F}}$ The conductance is given by the Landauer formula

$$
G=G_{0} \mathrm{~T}_{1} \mathrm{tt}^{\dagger}=G_{0} \sum_{n-1}^{N} T_{n}
$$

If the conductor is such that all $T_{n} \ll 1$ (e g, a high tunnel barner), one finds $P=$ $2 e|V| G \equiv P_{\text {Poisson }}$, corresponding to a Poisson distubution of the emitted electrons It has been demonstiated by Levitov and Lesovik [23] (see also Ref [24]) that the general formula (1 1 ) corresponds to a binomial (or Beinoull1) distıbution of the emitted electrons for each transmission eigenstate If some $T_{n}$ are near 1 (open channels), then the shot noise is reduced below $P_{\text {Poisson }}$ This implies that in a quantum point contact the shot noise is absent on the plateaus of conductance quantization and appears only at the steps between the plateaus [2] This effect has indeed been observed in experments [25-27] In a metallic, diffusive conductor, the $T_{n}$ ate either exponentially small or of order unity [12] This bimodal distribution is required by Ohm's law for the average conductance [28] and has been deived microscopically by Nazaiov [9] and by Altshuler et al [10] As a consequence of the bimodal distubution, the shot-noise power is reduced to one-third of the Poisson nowse [6]

It has been emphasized by Landauer [29], that Coulomb interactions may induce a further reduction of $P$ Here, we follow the quantum mechanical treatments in assuming noninteracting electrons, within the framework of the Boltzmann-Langevin approach We do include the effects of electrostatic potential fluctuations in Section 5

\section{Boltzmann-Langevin equation}

We begin by formulating the semiclassical kinetic theory $[16,17]$ We consider a conductor with a $d$-dimensional density of states connected by ideal leads to two 


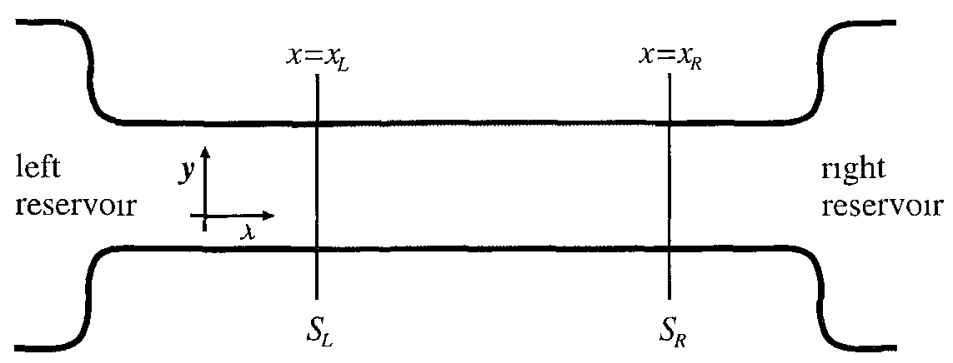

Fig 1 The conductor consists of a scattering region (dotted) connected by peifect leads to two election ieservons Cross sections $S_{\mathrm{L}}$ and $S_{\mathrm{R}}$ in the left and 11ght lead are indicated

electron reservolrs (see Fig 1) The ieservours have a temperature $T_{0}$ and a voltage difference $V$ The electrons in the left and the ingt reservorr are in equilibrium, with distribution function, $f_{\mathrm{L}}(\varepsilon)=f_{0}(\varepsilon-e V)$ and $f_{\mathrm{R}}(\varepsilon)=f_{0}(\varepsilon)$, respectively Heie $f_{0}$ is the Fermi-Dirac distribution,

$$
f_{0}(\varepsilon)=\left[1+\exp \left(\frac{\varepsilon-E_{\mathrm{F}}}{T_{0}}\right)\right]^{-1}
$$

The fluctuating distribution function $f(\mathbf{r}, \mathbf{k}, t)$ in the conductor equals $(2 \pi)^{d}$ times the density of electrons with position $\mathbf{r}$, and wave vector $\mathbf{k}$, at time $t$ [The factor $(2 \pi)^{d}$ is introduced so that $f$ is the occupation number of a unit cell in phase space ] The average over time-dependent fluctuations $\langle f\rangle \equiv \bar{f}$ obeys the Boltzmann equation,

$$
\begin{gathered}
\left(\frac{\mathrm{d}}{\mathrm{d} t}+\mathcal{S}\right) \bar{f}(\mathbf{r}, \mathbf{k}, t)=0, \\
\frac{\mathrm{d}}{\mathrm{d} t} \equiv \frac{\partial}{\partial t}+\mathbf{v} \frac{\partial}{\partial \mathbf{r}}+\mathcal{F} \frac{\partial}{\hbar \partial \mathbf{k}}
\end{gathered}
$$

The derivative (2 2b) (with $\mathbf{v}=\hbar \mathbf{k} / m$ ) describes the classical motion in the force field $\mathcal{F}(\mathbf{r})=-e \partial \phi(\mathbf{r}) / \partial \mathbf{r}+e \mathbf{v} \times \mathbf{B}(\mathbf{r})$, with electiostatic potential $\phi(\mathbf{r})$ and magnetic field $\mathbf{B}(\mathbf{r})$ The term $\mathcal{S} \bar{f}$ accounts for the stochastic effects of scattering Only elastic scattering is taken into account and electron--electron scattering is disiegarded In the case of impurity scattering, the scatteing term in the Boltzmann equation (22) is given by

$$
\begin{aligned}
\mathcal{S} f(\mathbf{r}, \mathbf{k}, t) & =\int \mathrm{d} \mathbf{k}^{\prime} W_{\mathbf{k} \mathbf{k}^{\prime}}(\mathbf{r})\left\{f(\mathbf{r}, \mathbf{k}, t)\left[1-f\left(\mathbf{r}, \mathbf{k}^{\prime}, t\right)\right]-f\left(\mathbf{r}, \mathbf{k}^{\prime}, t\right)[1-f(\mathbf{r}, \mathbf{k}, t)]\right\} \\
& =\int \mathrm{d} \mathbf{k}^{\prime} W_{\mathbf{k} \mathbf{k}^{\prime}}(\mathbf{r})\left[f(\mathbf{r}, \mathbf{k}, t)-f\left(\mathbf{r}, \mathbf{k}^{\prime}, t\right)\right]
\end{aligned}
$$

Here, $W_{\mathbf{k k}^{\prime}}(\mathbf{r})$ is the tiansition rate for scattering fiom $\mathbf{k}$ to $\mathbf{k}^{\prime}$, which may in pinciple also depend on $\mathbf{r}$ [We assume inversion symmetry, so that $W_{\mathbf{k k}^{\prime}}(\mathbf{r})=W_{\mathbf{k}^{\prime} \mathbf{k}}(\mathbf{r})$ ]

We consider the stationary situation, wheie $\bar{f}$ is independent of $t$ The time-dependent fluctuations $\delta f \equiv f-\bar{f}$ satisfy the Boltzmann-Langevin equation $[16,17]$,

$$
\left(\frac{\mathrm{d}}{\mathrm{d} t}+\mathcal{S}\right) \delta f(\mathbf{r}, \mathbf{k}, t)=J(\mathbf{r}, \mathbf{k}, t),
$$


where $j$ is a fluctuating source term representing the fluctuations induced by the stochastic natue of the scatteing The flux $y$ has zelo average, $\langle j\rangle=0$, and covariance

$$
\left\langle J(\mathbf{r}, \mathbf{k}, t) J\left(\mathbf{1}^{\prime}, \mathbf{k}^{\prime}, t^{\prime}\right)\right\rangle=(2 \pi)^{d} \delta\left(\mathbf{r}-\mathbf{r}^{\prime}\right) \delta\left(t-t^{\prime}\right) J\left(\mathbf{r}, \mathbf{k}, \mathbf{k}^{\prime}\right)
$$

The delta functions ensure that fluxes are only correlated if they are induced by the same scattering process The flux conelator $J$ depends on the type of scattering and on $\bar{f}$, but not on $\delta f$ The correlator $J$ for the impunity-scattening term (23) has been derived by Kogan and Shul'man [17],

$$
\begin{aligned}
J\left(\mathbf{r}, \mathbf{k} \mathbf{k}^{\prime}\right)= & -W_{\mathbf{k} \mathbf{k}}(\mathbf{r})\left[\bar{f}\left(1-\bar{f}^{\prime}\right)+\bar{f}^{\prime}(1-\bar{f})\right] \\
& +\delta\left(\mathbf{k}-\mathbf{k}^{\prime}\right) \int \mathrm{d} \mathbf{k}^{\prime \prime} W_{\mathbf{k k}}(\mathbf{r})\left[\bar{f}\left(1-\bar{f}^{\prime \prime}\right)+\bar{f}^{\prime \prime}(1-\bar{f})\right],
\end{aligned}
$$

wherc $\bar{f} \equiv \bar{f}(\mathbf{r}, \mathbf{k}), \bar{f}^{\prime} \equiv \bar{f}\left(\mathbf{r}, \mathbf{k}^{\prime}\right)$, and $\bar{f}^{\prime \prime} \equiv \bar{f}\left(\mathbf{r}, \mathbf{k}^{\prime \prime}\right)$ One veuffes that

$$
\int \mathrm{d} \mathbf{k} J\left(\mathbf{r}, \mathbf{k}, \mathbf{k}^{\prime}\right)=\int \mathrm{d} \mathbf{k}^{\prime} J\left(\mathbf{r}, \mathbf{k}, \mathbf{k}^{\prime}\right)=0,
$$

as it should, since the fluctuating source teim conserves the number of particles [ $\int \mathrm{d} \mathbf{k} J(\mathbf{r}, \mathbf{k}, t)=0$ ] For the derivation of Eq (26) we 1efer to Ref [17] In Section 4 we give a similar derivation for $J$ in the case of baitier scatteing

Since $y$ and $y^{\prime}$ ate unconclated for $t>t^{\prime}$, it follows from Eq (24) that the contelation function $\left\langle\delta f \delta f^{\prime}\right\rangle$ satisfies a Boltzmann equation in the valiables $\mathbf{r}, \mathbf{k}, t$,

$$
\left(\frac{\mathrm{d}}{\mathrm{d} t}+\mathcal{S}\right)\left\langle\delta f(\mathbf{r}, \mathbf{k}, t) \delta f\left(\mathbf{r}^{\prime}, \mathbf{k}^{\prime}, t^{\prime}\right)\right\rangle=0
$$

Eq (2 8) forms the statting point of the method of moments of Gantsevich et al [30] This method is vely convenient to study equilibium fluctuations, because the equal-time corselation is known,

$$
\left\langle\delta f(\mathbf{r}, \mathbf{k}, t) \delta f\left(\mathbf{r}^{\prime}, \mathbf{k}^{\prime}, t\right)\right\rangle_{\text {cquilibıum }}=(2 \pi)^{d} \bar{f}(\mathbf{r}, \mathbf{k})[1-\bar{f}(\mathbf{r}, \mathbf{k})] \delta\left(\mathbf{k}-\mathbf{k}^{\prime}\right) \delta\left(\mathbf{r}-\mathbf{r}^{\prime}\right),
$$

and $\mathrm{Eq} \mathrm{(2} \mathrm{8)} \mathrm{can} \mathrm{be} \mathrm{used} \mathrm{to} \mathrm{compute} \mathrm{the} \mathrm{non-equal-time} \mathrm{correlation} \mathrm{(For} \mathrm{a} \mathrm{study} \mathrm{of}$ theimal noise within this appioach, sce, for example, Ref [31] ) Out of equilibium, Eq (29) does not hold, except in the 1eservolis, and one has to retuin to the full Boltzmann-Langevin cquation (24) to determine the shot noise In patticula1, it is only in equilibilum that the equal-time conelation $\left\langle\delta f \delta f^{\prime}\right\rangle$ vanishes for $\mathbf{r} \neq \mathbf{1}^{\prime}, \mathbf{k} \neq \mathbf{k}^{\prime}$ Out of equilibilum, scattering conelates fluctuations $\partial f$ at different momenta and different points in space

To obtain the shot-noise power we compute the cuntent $I(t) \equiv \bar{I}+\delta I(t)$ through a cross-section $S_{\mathrm{R}}$ in the 11ght lead The average current $\bar{I}$ and the fluctuations $\delta I(t)$ are given by

$$
\bar{I}=\frac{e}{(2 \pi)^{d}} \int_{S_{\mathrm{R}}} \mathrm{d} \mathbf{y} \int \mathrm{d} \mathbf{k} v_{\lambda} \bar{f}(\mathbf{r}, \mathbf{k}),
$$




$$
\delta I(t)=\frac{e}{(2 \pi)^{d}} \int_{S_{\mathrm{R}}} \mathrm{d} \mathbf{y} \int \mathrm{d} \mathbf{k} v_{x} \delta f(\mathbf{r}, \mathbf{k}, t)
$$

We denote $\mathbf{r}=(x, \mathbf{y})$, with the $x$-coordinate along and $\mathbf{y}$ perpendicular to the wite (see Fig 1) The zero-frequency noise power is defined as

$$
P \equiv 2 \int_{-\infty}^{\infty} \mathrm{d} t\langle\delta I(t) \delta I(0)\rangle
$$

The formal solution of Eq (24) is

$$
\delta f(\mathbf{r}, \mathbf{k}, t)=\int_{-\infty}^{t} \mathrm{~d} t^{\prime} \int \mathrm{d} \mathbf{r}^{\prime} \int \mathrm{d} \mathbf{k}^{\prime} \mathcal{G}\left(\mathbf{r}, \mathbf{k}, \mathbf{r}^{\prime}, \mathbf{k}^{\prime}, t-t^{\prime}\right) \jmath\left(\mathbf{r}^{\prime}, \mathbf{k}^{\prime}, t^{\prime}\right),
$$

where the Green's function $\mathcal{G}$ is a solution of

$$
\left(\frac{\mathrm{d}}{\mathrm{d} t}+\mathcal{S}\right) \mathcal{G}\left(\mathbf{r}, \mathbf{k}, \mathbf{r}^{\prime}, \mathbf{k}^{\prime}, t\right)=\delta\left(\mathbf{r}-\mathbf{r}^{\prime}\right) \delta\left(\mathbf{k}-\mathbf{k}^{\prime}\right) \delta(t),
$$

such that $\mathcal{G}=0$ if $t<0$ The transmission probability $T(\mathbf{r}, \mathbf{k})$ is the probability that an electron at $(\mathbf{r}, \mathbf{k})$ leaves the wire through the right lead It is related to $\mathcal{G}$ by

$$
T(\mathbf{r}, \mathbf{k})=\int_{0}^{\infty} \mathrm{d} t \int_{S_{\mathrm{R}}} \mathrm{d} \mathbf{y}^{\prime} \int \mathrm{d} \mathbf{k}^{\prime} v_{x}^{\prime} \mathcal{G}\left(\mathbf{r}^{\prime}, \mathbf{k}^{\prime}, \mathbf{r}, \mathbf{k}, t\right)
$$

Substitution of Eqs (2 11) and (2 13) into Eq (2 12) yield for the notse power the expression

$$
\begin{aligned}
P= & \frac{2 e^{2}}{(2 \pi)^{2 d}} \int_{S_{\mathrm{R}}} \mathrm{d} \mathbf{y} \int \mathrm{d} \mathbf{k} v_{x} \int_{S_{\mathrm{R}}} \mathrm{d} \mathbf{y}^{\prime} \int \mathrm{d} \mathbf{k}^{\prime} v_{x}^{\prime} \\
& \times \int_{-\infty}^{t} \mathrm{~d} t^{\prime \prime} \int \mathrm{d} \mathbf{r}^{\prime \prime} \int \mathrm{d} \mathbf{k}^{\prime \prime} \mathcal{G}\left(\mathbf{r}, \mathbf{k}, \mathbf{r}^{\prime \prime}, \mathbf{k}^{\prime \prime}, t-t^{\prime \prime}\right) \\
& \times \int_{-\infty}^{0} \mathrm{~d} t^{\prime \prime \prime} \int \mathrm{d} \mathbf{r}^{\prime \prime \prime} \int \mathrm{d} \mathbf{k}^{\prime \prime \prime} \mathcal{G}\left(\mathbf{r}^{\prime}, \mathbf{k}^{\prime}, \mathbf{r}^{\prime \prime \prime}, \mathbf{k}^{\prime \prime \prime},-t^{\prime \prime \prime}\right) \\
& \times\left\langle J\left(\mathbf{r}^{\prime \prime}, \mathbf{k}^{\prime \prime}, t^{\prime \prime}\right) J\left(\mathbf{r}^{\prime \prime \prime}, \mathbf{k}^{\prime \prime \prime}, t^{\prime \prime \prime}\right)\right\rangle,
\end{aligned}
$$

which can be simplified using Eqs (2 5) and (2 15)

$$
P=\frac{2 e^{2}}{(2 \pi)^{d}} \int \mathrm{d} \mathbf{r} \int \mathrm{d} \mathbf{k} \int \mathrm{d} \mathbf{k}^{\prime} T(\mathbf{r}, \mathbf{k}) T\left(\mathbf{r}, \mathbf{k}^{\prime}\right) J\left(\mathbf{r}, \mathbf{k}, \mathbf{k}^{\prime}\right)
$$

Eq (2 17) applies generally to any conductor It contains the no1se due to the current fluctuations induced by the scattering processes inside the conductor At nonzero temperatures, there is an additional source of noise from fluctuations which otiginate from 
the reservoirs In Appendix A it is shown how this thermal noise can be incorporated In what follows, we restrict to zero temperature

A final remark concerns the $x$-coordinate of the cross-section at which the curient is evaluated [at $x=x_{\mathrm{R}}$ in Eq (2 11)] From current conservation it follows that the zerofrequency noise power should not depend on the specific value of $x$ This is explicitly proven in Appendix B, as a check on the consistency of the formalism

\section{Impurity scattering}

In this section we specialize to elastic impuity scattening in a conductor made of a material with a spherical Ferm suiface and in which the force field $\mathcal{F}=0$ (so we do not consider the case that a magnetic field is p1esent) The conductor has a length $L$ and a constant width $W(d=2)$ or a constant cross-sectional a1ea $A(d=3)$ (In general expressions, both $W$ and $A$ will be denoted by $A$ ) We calculate the shot noise at zero temperatuie and small applied voltage, $e V \ll E_{\mathrm{F}}$, so that we need to consider electrons at the Fermı energy only The case of nonzero temperature is briefly discussed in Appendix A

It 1 s useful to change variables from wave vector $\mathbf{k}$ to energy $\varepsilon=\hbar^{2} k^{2} / 2 m$, and unit vector $\hat{\mathbf{n}} \equiv \mathbf{k} / k$ The integrations are modified accordingly,

$$
\int \frac{\mathrm{d} \mathbf{k}}{(2 \pi)^{d}} \rightarrow \int \mathrm{d} \varepsilon \mathcal{D}(\varepsilon) \int \frac{\mathrm{d} \hat{\mathbf{n}}}{s_{d}},
$$

where $\mathcal{D}(\varepsilon)=s_{d} m(k / 2 \pi)^{d-2} h^{-2}$ is the density of states, and $s_{d}$ is the surface of a $d$-dimensional unit sphere $\left(s_{1}=2, s_{2}=2 \pi, s_{3}=4 \pi\right)$ We consider the case of specular boundary scatteing and assume that the elastic impurity-scatteing rate $W_{\mathbf{k k}}(\mathbf{r}) \equiv$ $W_{\mathbf{n n}^{\prime}} \delta\left(\varepsilon-\varepsilon^{\prime}\right) / \mathcal{D}(\varepsilon)$ is independent of $\mathbf{r}$ This allows us to drop the transverse coordinate $\mathbf{y}$ and write $T(\mathbf{r}, \mathbf{k})=T(x, \hat{\mathbf{n}})$ for the transmission piobability at the Fermi level From Eqs (2 14) and (2 15) we derive a Boltzmann equation for the transmission piobability $[15]$,

$$
v_{\mathrm{F}} n_{\lambda} \frac{\partial T(x, \hat{\mathbf{n}})}{\partial x}=\mathcal{S} T(x, \hat{\mathbf{n}})=\int \frac{\mathrm{d} \hat{\mathbf{n}}^{\prime}}{s_{d}} W_{\mathrm{nn}^{\prime}}\left[T(x, \hat{\mathbf{n}})-T\left(x, \hat{\mathbf{n}}^{\prime}\right)\right]
$$

The boundary conditions in the left and the ingt leads are

$$
\begin{aligned}
& T(0, \hat{\mathbf{n}})=0 \quad \text { if } n_{x}<0, \\
& T(L, \hat{\mathbf{n}})=1 \quad \text { if } n_{\lambda}>0,
\end{aligned}
$$

where $x_{\mathrm{L}}=0$ and $x_{\mathrm{R}}=L$ are the $x$-coordinates of the left and right cross-section $S_{\mathrm{L}}$ and $S_{\mathrm{R}}$, respectively

The average distribution function can be expressed as

$$
\bar{f}(\mathbf{r}, \mathbf{k})=[1-T(\mathbf{r},-\mathbf{k})] f_{\mathrm{L}}(\varepsilon)+T(\mathbf{r},-\mathbf{k}) f_{\mathrm{R}}(\varepsilon),
$$


where (because of time-reversal symmetry in the absence of a magnetic field) $T(\mathbf{r},-\mathbf{k}$ ) equals the probability that an electron at $(\mathbf{r}, \mathbf{k})$ has arrived there fiom the right 1eservoli Combining Eqs (2 10) and (3 3), we obtain the semiclassical Landauer formula for the linear-1esponse conductance $G \equiv \lim _{V \rightarrow 0} \bar{I} / V$ [32],

$$
\begin{aligned}
G & =\frac{e^{2}}{(2 \pi)^{d}} \int_{S} \mathrm{~d} \mathbf{y} \int \mathrm{d} \mathbf{k}\left(-\frac{\partial f_{0}(\varepsilon)}{\partial \varepsilon}\right) v_{\lambda} T(\mathbf{r}, \mathbf{k}) \\
& =e^{2} \int_{S} \mathrm{~d} \mathbf{y} \int \mathrm{d} \varepsilon \mathcal{D}(\varepsilon)\left(-\frac{\partial f_{0}(\varepsilon)}{\partial \varepsilon}\right) \int \frac{\mathrm{d} \hat{\mathbf{n}}}{s_{d}} v n_{\lambda} T(x, \hat{\mathbf{n}}) \\
& =G_{0} N \int \frac{\mathrm{d} \hat{\mathbf{n}}}{v_{d-1}} n_{\imath} T(x, \hat{\mathbf{n}}),
\end{aligned}
$$

with $S_{x}$ the cross-section at $x$ The number of transverse modes $N=v_{d-1}\left(k_{\mathrm{F}} / 2 \pi\right)^{d-1} A$, where $v_{d}$ is the volume of a $d$-dimensional unit sphere $\left(v_{0}=1, v_{1}=2, v_{2}=\pi\right)$ One has $N=k_{\Gamma} W / \pi$ for $d=2$ and $N=k_{\Gamma}^{2} A / 4 \pi$ for $d=3$ One can venfy that the conductance in Eq (34) is 1ndependent of the value of $x$, as it should By 1ntegrating Eq (32a) over $\hat{\mathbf{n}}$ one finds that

$$
\frac{\partial}{\partial x} \int \mathrm{d} \hat{\mathbf{n}} n_{x} T(x, \hat{\mathbf{n}})=0
$$

We evaluate the noise power by substitution of Eqs (2 6) and (3 3) into Eq (2 17) Some intermediate steps are given in Appendix A The resulting zero-temperature shotnoise powel is

$$
\begin{aligned}
P= & \frac{P_{0} N}{v_{\mathrm{F}} S_{d} v_{d-1}} \int_{0}^{L} \mathrm{~d} x \int \mathrm{d} \hat{\mathbf{n}} \int \mathrm{d} \hat{\mathbf{n}}^{\prime} W_{\mathbf{n n}}\left[T(x, \hat{\mathbf{n}})-T\left(x, \hat{\mathbf{n}}^{\prime}\right)\right]^{2} \\
& \times T(x,-\hat{\mathbf{n}})\left[1-T\left(x,-\hat{\mathbf{n}}^{\prime}\right)\right]
\end{aligned}
$$

This completes ou general semiclassical theory What iemains is to compute the transmission probabilities fiom Eqs (32) for a paiticular choice of the scatteing late $W$ Comparing Eqs (12) and (34), we note that $\sum_{n} T_{n}$ collesponds semiclassically to $N \int \mathrm{d} \hat{\mathbf{n}} n_{x} T(x, \hat{\mathbf{n}})$ Comparison of Eqs (1 1$)$ and (3 6) shows that the semiclassical correspondence to $\sum_{n} T_{n}\left(1-T_{n}\right)$ is much more complicated, as it involves the transmission probabilities $T(x, \hat{\mathbf{n}})$ at all scatterers inside the conductor (and not just the transmission probability $T(0, \hat{\mathbf{n}})$ thiough the whole conductor)

In a ballistic conductor, where impuity scattering is absent, the transmission piobabilities are given by $T(x, \hat{\mathbf{n}})=1$, if $n_{x}>0$, and $T(x, \hat{\mathbf{n}})=0$, if $n_{1}<0$ From Eq (34), we then obtain the Sharvin conductance $G_{\mathrm{S}} \equiv G_{0} N$ [33] Eq (36) 1mplies that the shot-noise power is zeio, in agreement with a previous semiclassical calculation by Kulık and Omel'yanchuk [18]

We now 1estrict ourselves to the case $W_{\mathrm{nn}}=v_{\Gamma} / \ell$ of isotiopic impuirty scatteing Let us first show that in the diffusive limit $(\ell \ll L)$ the result of Nagaev [7] is recovered 
Fo1 a diffusive wire the solution of Eq (3 4) can be appioximated by

$$
T(r, \hat{\mathbf{n}})=\frac{x+\ell n_{r}}{L}
$$

Deviations fiom this appioximation only occur within a thin layer, of order $\ell$, at the ends $\imath=0$ and $x=L$ Substitution of $\mathrm{Eq} \mathrm{(3} \mathrm{7)} \mathrm{into} \mathrm{Eq} \mathrm{(3} \mathrm{4)} \mathrm{yields} \mathrm{the} \mathrm{Drude}$ conductance

$$
G_{\mathrm{D}}=G_{0} N \frac{\tilde{\ell}}{L}
$$

with the noimalized mean fiee path $\tilde{\ell} \equiv\left(v_{d} / v_{d-1}\right) \ell$, i e for $d=2$ we have $\tilde{\ell}=\frac{1}{2} \pi \ell$ and for $d=3$ we have $\tilde{\ell}=\frac{4}{3} \ell$ For the shot-norse power we obtain fiom $\mathrm{Eq}(36)$, neglecting terms of orde1 $(\ell / L)^{2}$,

$$
P=P_{0} N \frac{2 \tilde{\ell}}{L} \int_{0}^{L} \frac{\mathrm{d} x}{L} \frac{x}{L}\left(1-\frac{x}{L}\right)=\frac{1}{3} P_{\text {Poisson }},
$$

1 agieement with Nagaev [7] This 1esult is a dilect consequence of the linear dependence of the transmission piobability (37) on $x$, which is geneic for diffusive transport In Appendix $C$ it is demonstrated that for a diffusive conductor with arbitrary (nonisotiop1c) impuity scattering $W_{\text {nn }}$, the 1esult $P=\frac{1}{3} P_{\text {Porsson }} 1$ ema1ns valid

We can go beyond Ref [7] and apply our method to quas1-ballistic conductors, for which $\ell$ and $L$ become comparable In Ref [15], we showed how in this case the probability $T(x, \hat{\mathbf{n}})$ can be calculated numerically by solving Eq (32) With this numeical solution as input, we compute the conductance and the shot-noise power fiom Eqs (34) and (36) The result is shown in Fig 2 The conductance ciosses over from the Sharvin conductance to the Diude conductance with increasing length [15] This crossover is accompanied by a i1se in the shot noise, from zero to $\frac{1}{3} P_{\text {Porson }}$ We note small differences between the two- and the three-dimensional case in the crossove regime The crossove is only weakly dependent on the dimensionality of the Fermi surface

The dimensions $d=2$ and 3 require a numerical solution of Eqs (32) Fol $d=1$ an analytical solution is possible We emphasize that this is not a model for true onedimensional tianspoit, whese quantum inteifeience leads to localization if $L>\ell$ [34] The case $d=1$ should iather be consideied as a toy model, which displays similaı behavior as the two and three-dimensional cases, but which allows us to evaluate both the conductance and the shot-noise power analytically for arbitiary ratio $\ell / L$ In the case $d=1$ an election can move either forward or backward, so $n_{\tau} \equiv n$ is either 1 or -1 The solution of Eq (32) is

$$
T(x, n)=\frac{x+\ell(n+1)}{L+2 \ell}
$$




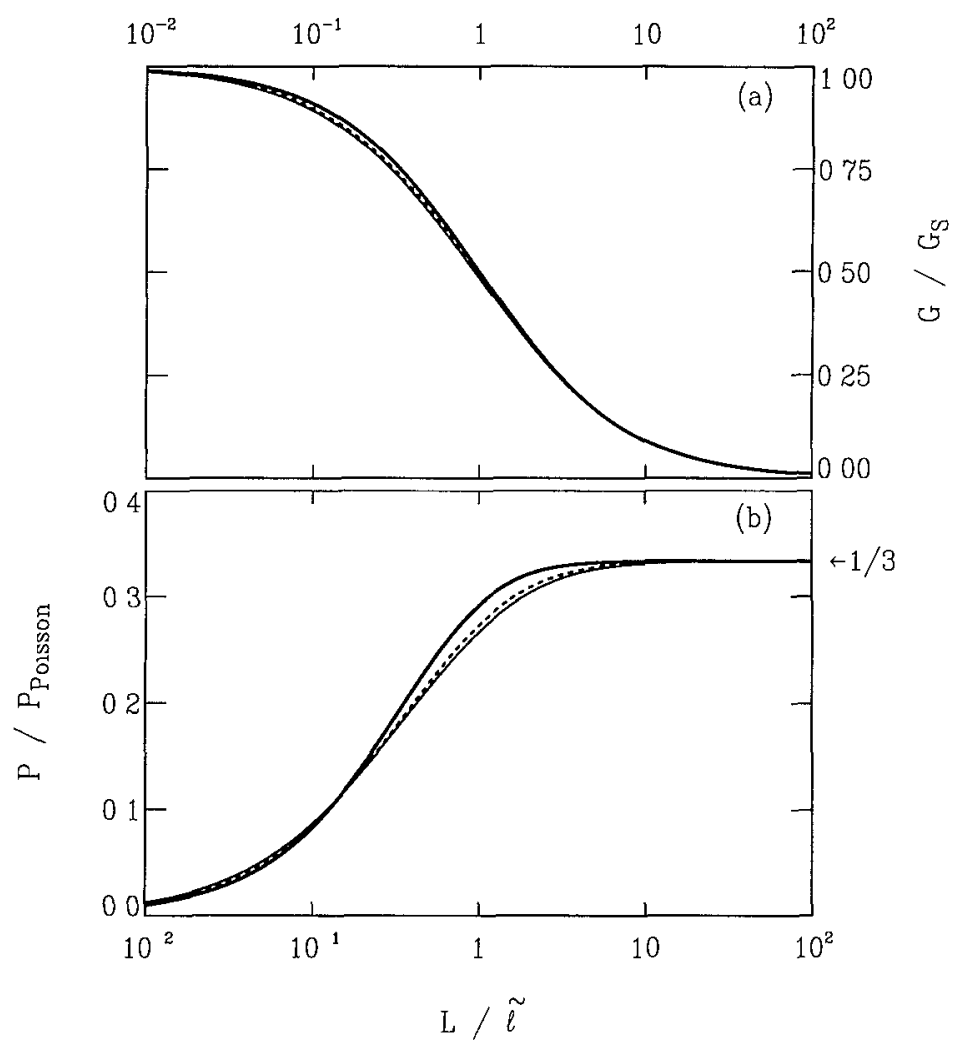

Fig 2 (a) The conductance (normalized by the Sharvin conductance $G_{S}=N G_{0}$ ) and (b) the shot-noise power (in units of $P_{\text {Poisson }} \equiv 2 e|\bar{I}|$ ), as a function of the ratio $L / \tilde{I}$ computed fiom Eqs (34) and (36) for isotiopic impuity scattering The curves coriespond to a three-dimensional (thin solid cuive), two dimenstonal (dashed curve), and a one-dimensional conductor (thick solid curve) The one-dimensional case is the analytical tesult from Eqs (3 11) and (312) The two- and three dimensional cases are numerical results

Substitution into Eq (3 4) yrelds

$$
G=G_{0} N \frac{1}{1+L / \tilde{\ell}},
$$

where $\tilde{\ell} \equiv 2 \ell$ Note that the resistance $1 / G$ is precisely the sum of the Drude and the Sharvin resistance The shot-noise power follows from Eq (36),

$$
P=P_{0} N \frac{\frac{2}{3} \ell L^{3}+4 \ell^{2} L^{2}+8 \ell^{3} L}{(L+2 \ell)^{4}}=\frac{1}{3}\left(1-\frac{1}{(1+L / \tilde{\ell})^{3}}\right) P_{\text {Polsson }}
$$

In Fig 2 we have plotted $G$ and $P$ according to Eqs (3 11) and (3 12) The difference between the results for $d=2$ and $d=3$ is very small

Liu et al [35] have carried out Monte Cailo simulations of the shot noise in a mesoscopic conductor, in good agreement with Eq (3 12) In Ref [8], we have peiformed a quantum mechanical study of the shot noise in a wire geometry, on the basis of 


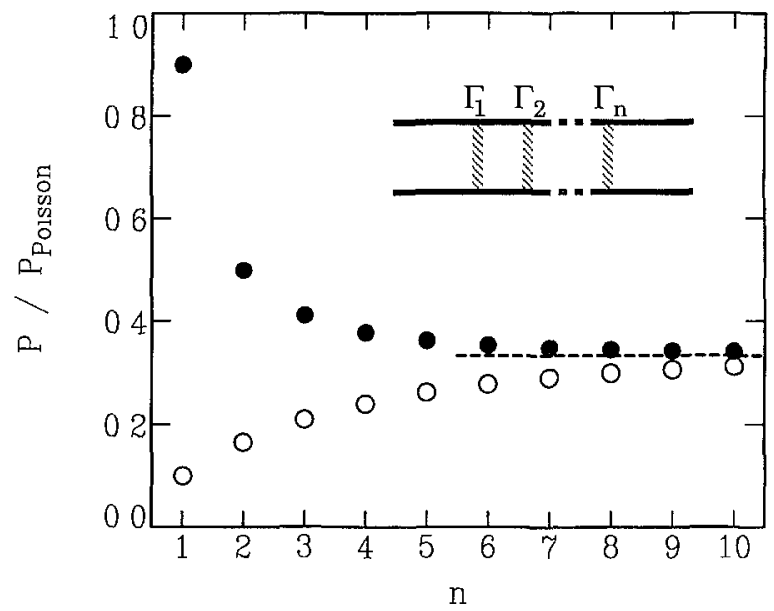

Fig 3 The shot noise power $P$ for $n$ tunnel baulers in selıes with transmission probability $\Gamma=01$ (dots) and $\Gamma=09$ (curcles), computed from $\mathrm{Eq}$ (4 10) The dashed line is the large- $n$ limit $P=\frac{1}{3} P_{\text {Poisson }}$ The inset shows schematically the geometry consideied

the Dorokhov-Mello-Pereyra-Kumat equation [36] The semiclassical results for $d=1$ obtained in the present paper, both for the conductance and for the shot-norse power, coincide piecisely with these quantum mechanical results, in the limit $N \tilde{\ell} / L \gg 1$ Cor1ections (of order $P_{0}$ ) to the shot-norse power, due to weak localization [8], are beyond the semiclassical approach

\section{Barrier scattering}

We now specialize to the case that the scattering is due to $n$ planar tunnel baniers in seives, perpendicular to the $x$-dilection (see inset of Fig 3) Barriel $l$ has tunnel piobability $\Gamma_{I} \in[0,1]$, which for simplicity is assumed to be $\mathbf{k}$ and $\mathbf{y}$-independent In what follows, we again drop the $\mathbf{y}$-coordinate Upon transmission $\mathbf{k}$ is conselved, whereas upon reflection $\mathbf{k} \rightarrow \widetilde{\mathbf{k}} \equiv\left(-k_{\imath}, \mathbf{k}_{\jmath}\right.$ ) At barries $\imath$ (at $x=x_{1}$ ) the average densities $\bar{f}$ on the left side $\left(x_{l-}\right)$ and on the right side $\left(x_{l+}\right)$ are related by

$$
\begin{array}{ll}
\bar{f}\left(x_{l+}, \mathbf{k}\right)=\Gamma_{l} \bar{f}\left(x_{l-}, \mathbf{k}\right)+\left(1-\Gamma_{l}\right) \bar{f}\left(x_{l+}, \widetilde{\mathbf{k}}\right) \text { if } k_{x}>0, \\
\bar{f}\left(x_{l-}, \mathbf{k}\right)=\Gamma_{l} \bar{f}\left(x_{l+}, \mathbf{k}\right)+\left(1-\Gamma_{l}\right) \bar{f}\left(x_{l-}, \widetilde{\mathbf{k}}\right) \text { if } k_{\mathrm{r}}<0
\end{array}
$$

To determine the conelator $J$ in Eq (2 5), we argue in a similar way as in Ref [5] Consider an incoming state from the left $\left(x_{l-}, \mathbf{k}\right)$ and from the right $\left(x_{t+}, \widetilde{\mathbf{k}}\right)$ (we assume $\left.k_{x}>0\right)$ We need to distinguish between foul diffeient situations

(a) Both incoming states empty, probability $\left[1-\bar{f}\left(x_{t-}, \mathbf{k}\right)\right]\left[1-\bar{f}\left(x_{t+}, \widetilde{\mathbf{k}}\right)\right]$ Since no fluctuations in the outgoing states are possible, the contisution to $J$ is zero

(b) Both incoming states occupied, probability $\bar{f}\left(x_{l_{-}}, \mathbf{k}\right) \bar{f}\left(x_{i+}, \widetilde{\mathbf{k}}\right)$ Again, no contribution to $J$ 
(c) Incoming state from the left occupied and from the right empty, probability $\bar{f}\left(x_{l-}, \mathbf{k}\right)\left[1-\bar{f}\left(x_{l+}, \widetilde{\mathbf{k}}\right)\right]$. On the average, the outgoing states at the left and right have occupation $1-\Gamma_{l}$ and $\Gamma_{l}$, respectively. However, since the incoming electron is either transmitted or reflected, the instantaneous occupation of the outgoing states differs from the average occupation. Upon transmission, the state at the right (left) has an excess (deficit) occupation of $1-\Gamma_{l}$. Upon reflection, the state at the right (left) has a deficit (excess) occupation of $\Gamma_{l}$. Since transmission occurs with probability $\Gamma_{l}$ and reflection with probability $1-\Gamma_{l}$, the equal-time correlation of the occupations is given by

$$
\left\langle\delta f(\mathbf{r}, \mathbf{k}, t) \delta f\left(\mathbf{r}^{\prime}, \mathbf{k}^{\prime}, t\right)\right\rangle=\left\{\begin{array}{c}
(2 \pi)^{d} \Gamma_{l}\left(1-\Gamma_{l}\right) \delta\left(\mathbf{k}-\mathbf{k}^{\prime}\right) \delta\left(\mathbf{r}-\mathbf{r}^{\prime}\right) \\
\text { if } x, x^{\prime}>x_{l} \text { or } x, x^{\prime}<x_{l} \\
-(2 \pi)^{d} \Gamma_{l}\left(1-\Gamma_{l}\right) \delta\left(\mathbf{k}-\widetilde{\mathbf{k}}^{\prime}\right) \delta\left(\mathbf{y}-\mathbf{y}^{\prime}\right) \delta\left(x+x^{\prime}-2 x_{l}\right) \\
\text { if } x<x_{l}<x^{\prime} \text { or } x^{\prime}<x_{l}<x .
\end{array}\right.
$$

In terms of the fluctuating source, the fluctuating occupation number can be expressed as

$$
\delta f(\mathbf{r}, \mathbf{k}, t)=\frac{1}{\left|v_{\imath}\right|} \int \mathrm{d} x_{0} j\left(x_{0}, \mathbf{y}-\frac{\mathbf{v}_{y}}{v_{x}}\left(x-x_{0}\right), \mathbf{k}, t-\frac{x-x_{0}}{v_{\lambda}}\right),
$$

where we have used Eq. (2.13). (This result is valid as long as only one scattering event has occurred.) Combining Eqs. (4.2) and (4.3), it is found that

$$
\begin{aligned}
\left\langle j(\mathbf{r}, \mathbf{k}, t) j\left(\mathbf{r}^{\prime}, \mathbf{k}^{\prime}, t^{\prime}\right)\right\rangle= & (2 \pi)^{d} \Gamma_{l}\left(1-\Gamma_{l}\right) \delta\left(x-x_{l}\right)\left|v_{\imath}\right| \delta\left(\mathbf{r}-\mathbf{r}^{\prime}\right) \\
& \times\left[\delta\left(\mathbf{k}-\mathbf{k}^{\prime}\right)-\delta\left(\mathbf{k}-\widetilde{\mathbf{k}}^{\prime}\right)\right] \delta\left(t-t^{\prime}\right),
\end{aligned}
$$

upon the initial condition of occupied left and unoccupied right incoming state.

(d) For an incoming state from the left unoccupied and from the right occupied, the probability is $\left[1-\bar{f}\left(x_{1-}, \mathbf{k}\right)\right] \bar{f}\left(x_{t+}, \widetilde{\mathbf{k}}\right)$. Similar to situation (c).

Collecting results from (a)-(d) and summing over all barriers, we find

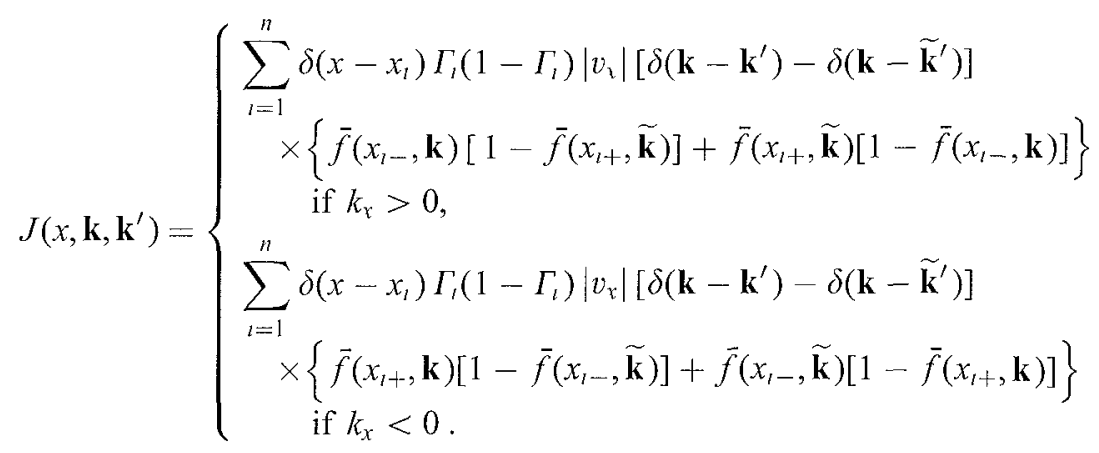

Substitution of Eqs. (3.3) and (4.5) into Eq. (2.17) and linearization in $V$ yields

$$
P=P_{0} N \sum_{l=1}^{n} \Gamma_{l}\left(1-\Gamma_{l}\right)\left(T_{i}^{\rightarrow}-T_{l}^{\leftarrow}\right)^{2}\left(T_{l}^{\rightarrow}+T_{l}^{\leftarrow}-2 T_{l}^{\rightarrow} T_{l}^{\leftarrow}\right),
$$


where $T_{l} \rightarrow \equiv T\left(x_{l+}, k_{\mathrm{r}}>0\right)\left[T_{l}^{\leftarrow} \equiv T\left(x_{i}, k_{\lambda}<0\right)\right]$ is the transmission probability into the right 1eservorr of an election at the Fermı level moving away from the right [left] side of banies $l$ The conductance is given simply by

$$
G=G_{0} N T
$$

where $T \equiv T\left(x_{1}, k_{\lambda}>0\right)$ is the transmission probability through the whole conductor

As a first application of $\mathrm{Eq}$ (46), we calculate the shot noise for a single tunnel banter Using $T=\Gamma, T_{1}^{\leftarrow}=0, T_{1}=1$, we find the expected result [1 5$] P=P_{0} N \Gamma(1-$ $\Gamma)=(1-\Gamma) P_{\text {Poisson }}$ The double-bauner case $(n=2)$ is less tuvial Expenments by L1 et al [37] and by Liu et al [38] showed full Poisson noise, for asymmetnc stiuctures $\left(\Gamma_{1} \ll \Gamma_{2}\right)$ and a suppiession by one half, for the symmetıc case $\left(\Gamma_{1} \simeq \Gamma_{2}\right)$ This effect has been explained by Chen and Ting [21], by Davies et al [22], and by others [39] These theorles assume resonant tunneling in the regime that the applied voltage $V$ is much gieatel than the width of the resonance This requiles $\Gamma_{1}, \Gamma_{2} \ll 1$ The present semiclassical appioach makes no ieference to tiansmission resonances and is valid for all $\Gamma_{1}, \Gamma_{2}$ For the double-ban1er system one has $T=\Gamma_{1} \Gamma_{2} / \Delta, T_{1}^{-}=0, T_{1}=\Gamma_{2} / \Delta$, $T_{2}^{\leftarrow}=\left(1-\Gamma_{1}\right) \Gamma_{2} / \Delta$, and $T_{2}^{\rightarrow}=1$, with $\Delta=\Gamma_{1}+\Gamma_{2}-\Gamma_{1} \Gamma_{2}$ Fiom Eqs (4 6) and (4 7), it follows that

$$
P=\frac{\Gamma_{1}^{2}\left(1-\Gamma_{2}\right)+\Gamma_{2}^{2}\left(1-\Gamma_{1}\right)}{\left(\Gamma_{1}+\Gamma_{2}-\Gamma_{1} \Gamma_{2}\right)^{2}} P_{\text {Poisson }}
$$

In the limit $\Gamma_{\mathrm{I}}, \Gamma_{2} \ll 1, \mathrm{Eq}$ (4 8) coincides precisely with the results of Refs [21,22]

The shot-noise suppiession of one half for a symmetıc double-banier junction has the same oilgin as the one-thit suppiession for a diffusive conductor In our semiclassical model, this is evident fiom the fact that a diffusive conductor is the continuum limit of a senes of tunncl ban1ers We demonstrate this below Quantum mechancally, the common oilgin is the bimodal distibution $\rho(T) \equiv\left\langle\sum_{n} \delta\left(T-T_{n}\right)\right\rangle$ of transmission e1genvalues, which for a double-ban1e1 junction is given by [40]

$$
\rho(T)=\frac{N \Gamma_{1} \Gamma_{2}}{\pi T \sqrt{4 \Gamma_{1} \Gamma_{2} T-\left(4 T+\Gamma_{1} \Gamma_{2}\right)^{2}}},
$$

for $T \in\left[T_{-}, T_{+}\right]$, with $T_{ \pm}=\Gamma_{1} \Gamma_{2} /(1 \mp \sqrt{1-\Delta})^{2}$ For a symmetnc junction $\left(I_{1}=\Gamma_{2} \ll\right.$ 1), the density (49) 1s strongly peaked neat $T=0$ and $T=1$, leading to a suppiession of shot noise, just as in the case of a diffusive conductor In fact, one can veufy that the average of Eqs (11) and (12), with the bimodal distibution (49), gives precisely the result (4 8) fiom the Boltzmann Langevin equation

We now consider $n$ batuets with equal $\Gamma$ We find $T=\Gamma / \Delta, T_{r} \rightarrow=[\Gamma+\imath(1-\Gamma)] / \Delta$, and $T_{r}^{\leftarrow}=(t-1)(1-\Gamma) / \Delta$, with $\Delta=\Gamma+n(1-\Gamma)$ Substitution into Eqs (4 6) and (4 7$)$ yields

$$
P=\frac{1}{3}\left(1+\frac{n(1-\Gamma)^{2}(2+\Gamma)-\Gamma^{3}}{[\Gamma+n(1-\Gamma)]^{3}}\right) P_{\text {Polsson }}
$$


The shot-noise suppression for a low barrier $(\Gamma=09)$ and for a high barrier $(\Gamma=01)$ is plotted against $n$ in Fig 3 For $\Gamma=01$ we observe almost full shot norse if $n=1$, one-half suppression if $n=2$, and on increasing $n$ the suppression rapidly reaches onethird For $\Gamma=09$, we observe that $P / P_{\text {Poisson }}$ increases from almost zero to one-thind It is clear from $\mathrm{Eq}(410)$ that $P \rightarrow \frac{1}{3} P_{\text {Poisson }}$ for $n \rightarrow \infty$ independent of $\Gamma$

We can make the connection with elastic impuity scatteing in a disordered wire as follows The scattering occuis throughout the whole wire instead of at a discrete number of barriers For the semiclassical evaluation we thus take the limit $n \rightarrow \infty$ and $\Gamma \rightarrow 1$, such that $n(1-\Gamma)=L / \tilde{\ell}$ For the conductance and the shot-nose powe1 one then obtains from Eqs (47) and (4 10) exactly the same 1esults, Eqs (3 11) and (3 12), as for impurity scattering with a one-dimensional density of states This equivalence is expected, since in the one-dimensional model elections move exthei forward or backward, whereas in the model of $n$ planar tunnel barriers in series the transverse component of the wave vector becomes irrelevant

We conclude this section by considering a wire consisting of a disordered region, between $x=0$ and $x=L$ with mean free path $\ell$, in serles with a barrier, at $x=x_{\mathrm{b}}>L$ with transparency $\Gamma$ For analytical conventence, we study the one-dimensional case $d=1$ (We have seen earlier that the dependence on $d$ is quite weak ) By modifying Eqs (3 2) and (4 1), we find

$$
\begin{aligned}
& T(x, n)= \begin{cases}\frac{x+\ell(1+n)}{L \Gamma+2 \ell} \Gamma & \text { if } x \in[0, L], \\
T(x, L) & \text { if } x \in\left[L, x_{\mathrm{b}}\right),\end{cases} \\
& T(x,-1)=1-\frac{2 \ell \Gamma}{L \Gamma+2 \ell} \quad \text { if } x>x_{\mathrm{b}}, \\
& T(x, 1)=1 \quad \text { if } x>x_{\mathrm{b}}
\end{aligned}
$$

The conductance is given by Eq (34),

$$
G=G_{0} N \frac{\Gamma}{1+\Gamma L / \tilde{\ell}}
$$

The total resistance is thus the sum of the Drude resistance $R_{\mathrm{D}}=L / G_{0} N \tilde{\ell}$ and the barrier resistance $R_{\Gamma}=1 / G_{0} N \Gamma$ Combining Eqs (36) and (46), we obtain for the shot-noise power

$$
\begin{aligned}
P= & \frac{P_{0} N}{2 \ell} \int_{0}^{L} \mathrm{~d} x[T(x, 1)-T(x,-1)]^{2}[T(x, 1)+T(x,-1)-2 T(x, 1) T(x,-1)] \\
& +P_{0} N \Gamma(1-\Gamma)\left[T\left(x_{\mathrm{b}+}, 1\right)-T\left(x_{\mathrm{b}-},-1\right)\right]^{2} \\
& \times\left[T\left(x_{\mathrm{b}+}, 1\right)+T\left(x_{\mathrm{b}-},-1\right)-2 T\left(x_{\mathrm{b}+}, 1\right) T\left(x_{\mathrm{b}-},-1\right)\right]
\end{aligned}
$$




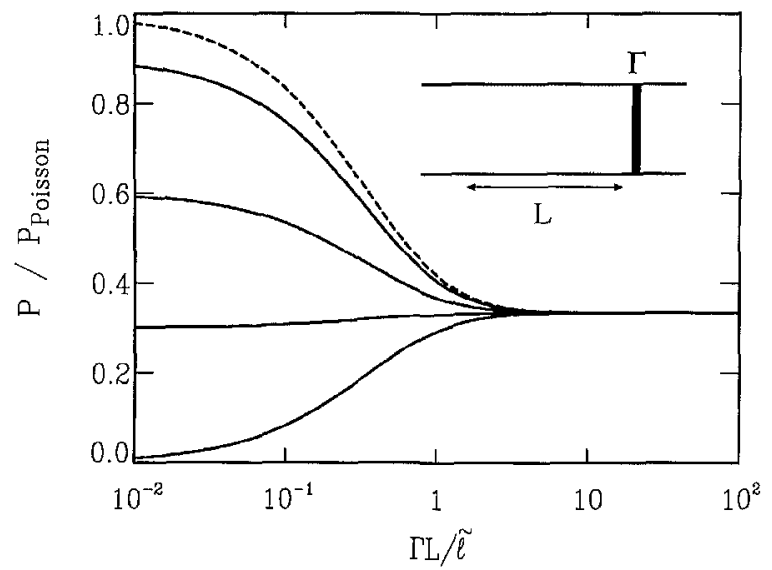

Fig. 4. The shot-noise power $P$ for a conductor consisting of a disordered region in series with a planar tunnel barrier (see inset) as a function of its length $L$ (in units of $\tilde{\ell} / \Gamma$ ), for barrier transparencies $\Gamma=1,0.7$, 0.4 , and 0.1 (bottom to top). The dashed line is the limiting curve for $\Gamma \ll 1$. The curves are computed from Eq. (4.14) for a model with a one-dimensional density of states. The dimensionality dependence is expected to be small, compare Fig. 2.

Substitution of Eqs. (4.11) yields

$$
\begin{aligned}
P & =P_{0} N\left(\frac{2 \Gamma^{3} \ell L\left(12 \ell^{2}+6 \ell L+\Gamma L^{2}\right)}{3(2 \ell+\Gamma L)^{4}}+\frac{8 \Gamma(1-\Gamma) \ell^{3}}{(2 \ell+\Gamma L)^{3}}\right) \\
& =\left(\frac{1}{3}-\frac{1}{3(1+\Gamma L / \tilde{\ell})^{3}}+\frac{1-\Gamma}{(1+\Gamma L / \tilde{\ell})^{3}}\right) P_{\text {Poisson }},
\end{aligned}
$$

where we have used Eq. (4.12). In Fig. 4 we have plotted the shot-noise power against the length of the disordered region for various values of the barrier transparency. In the absence of disorder, there is full shot noise for high barriers $(I \ll 1)$ and complete suppression if the barrier is absent $(\Gamma=1)$. Upon increasing the disorder strength, we note that the shot-noise power approaches the limiting value $P=\frac{1}{3} P_{\text {Poisson }}$ independent of $\Gamma$ : once the disordered region dominates the resistance, the shot noise is suppressed by one-third. Note that it follows from Eq. (4.14) that for $\Gamma=\frac{2}{3}$ the suppression is one-third for all ratios $\tilde{\ell} / L$.

We have carried out a quantum mechanical calculation of the shot-noise power in a wire geometry similar to the calculation in Ref. [8]. The barrier can be incorporated in the Dorokhov-Mello-Pereyra-Kumar equation [36] by means of an initial condition (see Ref. [41]). We find exactly the same result as Eq. (4.14) in the regime $N I \gg 1$ and $N \tilde{\ell} / L \gg 1$. For a high barrier $(I \ll 1)$ in series with a diffusive wire $(L \gg \tilde{\ell})$ our results for the shot noise coincide with previous work by Nazarov [9] using a different quantum mechanical theory. In this limit, the shot noise can be expressed as [9]

$$
P=\frac{1}{3}\left[1+2\left(\frac{R_{\Gamma}}{R}\right)^{3}\right] P_{\text {Poisson }}
$$


with the total resistance $R=R_{\mathrm{D}}+R_{\Gamma}$ The limiting result (4 15) 1s depicted by the dashed cuive in Fig 4

\section{Inelastic and electron-electron scattering}

In the previous sections we have calculated the shot noise for several types of elastic scattering In an experıment, however, additional types of scattering may occur In particular, electron-electron and inelastic election-phonon scatteing will be enhanced due to the high currents which are often requined for noise experiments The purpose of this section is to discuss the effects of these additional scatteing processes As shown by Nagaev [42] and by Kozub and Rudin [43], this can be achieved by including additional scattering terms in the Boltzmann-Langevin equation Here, we will adopt a different method, following Beenakkeı and Buttıkeı [6], in which inelastıc scatteıng is modeled by dividing the conductor in separate, phase-coherent parts which ate connected by charge-conserving ieservoirs We extend this model to include the following types of scattering

(a) Quast-elastic scattering Due to weak coupling with extennal degiees of fieedom the election wave function gets dephased, but its energy is conserved In metals, this scattering is caused by fluctuations in the electiomagnetic field [44]

(b) Electron heating Electron-election scattering exchanges energy between the elections, but the total energy of the election system is conserved The distibution function is therefore assumed to be a Fermi-Diac distıbution at a temperatue above the lattice tempcratuie

(c) Inelastic scattering Due to election phonon interactions the elections exchange energy with the lattice The electrons emeiging from the 1eservol1 a1e distributed according to the Fermi-Duac distribution (2 1), at the lattice temperature $T_{0}$ This is the model of Ref [6]

First, we divide the conductor in two pat ts connected via one reservoli and determine the shot norse for cases (a), (b) and (c) After that, we 1epeat the calculation for many intermediate 1eservors to take into account that the scatten occurs throughout the whole length of the conductor

The model is depicted in Fig 5 The conductors 1 and 2 are connected via a reservoli with disti1bution function $f_{12}(\varepsilon)$ The time-averaged cuirent $I_{m}$ through conductor $m=1,2$ is given by

$$
I_{1}=\left(G_{1} / e\right) \int \mathrm{d} \varepsilon\left[f_{\mathrm{L}}(\varepsilon)-f_{12}(\varepsilon)\right], \quad I_{2}=\left(G_{2} / e\right) \int \mathrm{d} \varepsilon\left[f_{12}(\varepsilon)-f_{\mathrm{R}}(\varepsilon)\right]
$$

The conductance $G_{m} \equiv 1 / R_{m}$ is expressed in terms of the transmission matrix $\mathrm{t}_{m}$ of conductor $m$ at the Ferm1 energy,

$$
G_{m}=G_{0} \operatorname{Tr} \mathbf{t}_{m} \mathbf{t}_{m}^{\dagger}=G_{0} \sum_{n-1}^{N} T_{n}^{(m)},
$$




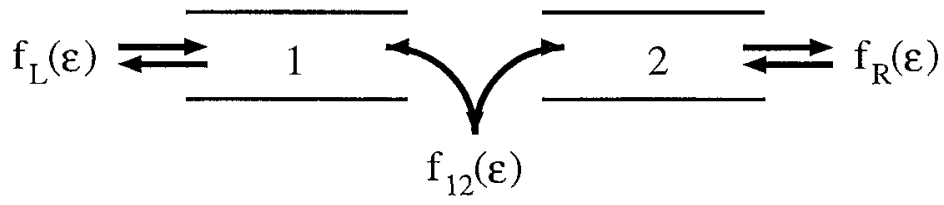

Fig. 5. Both ends of the conductor are connected to an electron rescrvorr. Addıtional scattering insıde the conductor is modeled by dividing it in two parts and connecting them through another reservorr. The electron distributions in the left and the right reservoir, $f_{\mathrm{L}}(\varepsilon)$ and $f_{\mathrm{R}}(\varepsilon)$, are Fermi-Dirac distributions. The distribution $f_{12}(\varepsilon)$ in the intermediate reservoir depends on the type of scatterng.

with $T_{n}^{(m)} \in[0,1]$ an eigenvalue of $\mathrm{t}_{m} \mathrm{t}_{m}^{\dagger}$. We assume small $\mathrm{eV}$ and $k_{\mathrm{B}} T_{0}$, so that we can neglect the energy dependence of the transmission eigenvalues.

Current conservation requires that

$$
I_{1}=I_{2} \equiv I
$$

We define the total resistance of the conductor by

$$
R=R_{1}+R_{2} \text {. }
$$

It will be shown that this incoherent addition of resistances is valid for all three types of scattering that we consider. Our model is not suitable for transport in the ballistic regime or in the quantum Hall regime, where a different type of "one-way" reservoirs are required [45]. Recently, Büttiker has calculated the effects of inelastic scattering along these lines [46].

The time-averaged current (5.1) depends on the average distribution $f_{12}(\varepsilon)$ in the reservoir between conductors 1 and 2 . In order to calculate the current fluctuations, we need to take into account that this distribution varies in time. We denote the timedependent distribution by $\tilde{f}_{12}(\varepsilon, t)$. The fluctuating current through conductor 1 or 2 causes electrostatic potential fluctuations $\delta \phi_{12}(t)$ in the reservoir, which enforce charge neutrality. In Ref. [6], the reservoir has a Fermi-Dirac distribution $\tilde{f}_{12}(\varepsilon, t)=f_{0}[\varepsilon-$ $\left.e V_{12}-e \delta \phi_{12}(t)\right]$, with $E_{\mathrm{F}}+e V_{12}$ the average electrochemical potential in the reservoir. As a result, it is found that the shot-noise power $P$ of the entire conductor is given by [6]

$$
R^{2} P=R_{1}^{2} P_{1}+R_{2}^{2} P_{2}
$$

In other words, the voltage fluctuations add. The noise powers $P_{1}$ and $P_{2}$ of the two segments depend solely on the time-averaged distribution [4],

$$
\begin{aligned}
& P_{1}=2 G_{1} \int \mathrm{d} \varepsilon\left[f_{\mathrm{L}}\left(1-f_{\mathrm{L}}\right)+f_{12}\left(1-f_{12}\right)\right]+2 S_{1} \int \mathrm{d} \varepsilon\left(f_{\mathrm{L}}-f_{12}\right)^{2}, \\
& P_{2}=2 G_{2} \int \mathrm{d} \varepsilon\left[f_{12}\left(1-f_{12}\right)+f_{\mathrm{R}}\left(1-f_{\mathrm{R}}\right)\right]+2 S_{2} \int \mathrm{d} \varepsilon\left(f_{12}-f_{\mathrm{R}}\right)^{2} .
\end{aligned}
$$


Here, $S_{m}$ is defined as

$$
S_{m}=G_{0} \operatorname{Tr} \mathbf{t}_{m} \mathbf{t}_{m}^{\dagger}\left(1-\mathbf{t}_{m} \mathbf{t}_{m}^{\dagger}\right)=G_{0} \sum_{n=1}^{N} T_{n}^{(m)}\left(1-T_{n}^{(m)}\right)
$$

For example, for a single tunnel barrier we have $S_{m}=G_{m}$, whereas for a diffusive conductor $S_{m}=\frac{1}{3} G_{m}$ The analysis of Ref [6] 1s easily generalized to atbitialy distribution $f_{12}$ Then, we have $\tilde{f}_{12}(\varepsilon, t)=f_{12}\left[\varepsilon-e \delta \phi_{12}(t)\right]$ It follows that Eqs (5 5) and (56) remain valid, but $f_{12}(\varepsilon)$ may be different Let us determine the shot noise for the three types of scattering

(a) Quast-elastic scattering Here, it is not just the total current which must be conserved, but the cuisent in each eneigy range This requires

$$
f_{12}(\varepsilon)=\frac{G_{1} f_{\mathrm{L}}(\varepsilon)+G_{2} f_{\mathrm{R}}(\varepsilon)}{G_{1}+G_{2}}
$$

We note that Eq (5 8) implies the validity of Eq (54) Substitution of Eq (5 8) into Eqs (5 5) and (56) yrelds at zero temperature the result

$$
P=P_{\text {Polsson }}\left(R_{1}^{4} S_{1}+R_{2}^{4} S_{2}+R_{1} R_{2}^{2}+R_{1}^{2} R_{2}\right) R^{-3}
$$

Fo1 a double-barrie1 junction in the limit $\Gamma_{1}, \Gamma_{2} \ll 1$, Eqs (4 8) and (5 9) give the same result, demonstrating that dephasing between the barries does not influence the shot noise This is in contiast to the result of Ref [47], where dephasing is modeled by adding random phases to the wave function For the diffusive wile Eq (5 9) implies $P=\frac{1}{3} P_{\text {Poisson, }}$, independent of the rat1o between $R_{1}$ and $R_{2}$ Breaking phase coherence, but retaining the nonequilibrium electron distribution leaves the shot noise unaltered The reservoir model for phase-breaking scattering is therefore consistent with the results of the Boltzmann-Langevin approach

(b) Electron heating We model electron-electron scattening, where energy can be exchanged between the elections, at constant total energy We assume that the exchange of energies establishes a Fermi-Dirac disti1bution $f_{12}(\varepsilon)$ at an electrochemical potential $E_{\mathrm{F}}+e V_{12}$ and an elevated temperature $T_{12}$ From current conservation, Eq (53), it follows that

$$
V_{12}=\frac{R_{2}}{R} V
$$

Conservation of the energy of the electron system requires that $T_{12}$ is such that no eneigy is absorbed or emitted by the reservorr The energy current $J_{m}$ thiough conductor $m$ is given by

$$
J_{1}=\left(\frac{G_{1}}{e^{2}}\right) \int \mathrm{d} \varepsilon \varepsilon\left[f_{\mathrm{L}}(\varepsilon)-f_{12}(\varepsilon)\right], \quad J_{2}=\left(\frac{G_{2}}{e^{2}}\right) \int \mathrm{d} \varepsilon \varepsilon\left[f_{12}(\varepsilon)-f_{\mathrm{R}}(\varepsilon)\right]
$$


Since $f_{12}$ is a Ferm1-Dirac distribution, Eq (5 11) equals

$$
\begin{aligned}
& J_{1}=Q_{1}+\mu_{1} I / e=K_{1}\left(T_{0}-T_{12}\right)+\mu_{1} I / e, \\
& J_{2}=Q_{2}+\mu_{2} I / e=K_{2}\left(T_{12}-T_{0}\right)+\mu_{2} I / e,
\end{aligned}
$$

where $\mu_{1} \equiv E_{\mathrm{F}}+\frac{1}{2} e\left(V+V_{12}\right)$ and $\mu_{2} \equiv E_{\mathrm{F}}+\frac{1}{2} e V_{12}$ The energy current $J_{m}$ is thus the sum of the heat current $Q_{m}$ and of the particle cunent $I / e$ times the average energy $\mu_{m}$ of each electron The heat current $Q_{m}$ equals the difference in temperature times the theimal conductance $K_{m}=G_{m} \mathcal{L}_{0} T_{m}$, with $T_{m} \equiv \frac{1}{2}\left(T_{0}+T_{12}\right)$ and the Lorentz number $\mathcal{L}_{0} \equiv \frac{1}{3}\left(k_{\mathrm{B}} / e\right)^{2} \pi^{2}$ There are no thermo-electric contributions in Eqs (51) and (5 12), because of the assumption of eneigy independent tiansmission eigenvalues [48] Fiom the requilement of eneigy conservation, $J_{1}=J_{2}$, we calculate the electron temperature in the intermediate 1eservol

$$
T_{12}^{2}=T_{0}^{2}+\frac{V^{2}}{\mathcal{L}_{0}} \frac{R_{1} R_{2}}{R^{2}}
$$

At ze1o temperature in the left and 11ght 1eservoll and for $R_{1}=R_{2}$ we have $k_{\mathrm{B}} T_{12}=$ $(\sqrt{3} / 2 \pi) e|V| \simeq 028 e|V|$ For the shot noise at $T_{0}=0$, we thus obtain using Eqs (5 5) and $(56)$,

$$
\begin{aligned}
P= & 2 \frac{k_{\mathrm{B}} T_{12}}{R}+2 \frac{S_{1} R_{1}^{2}}{R^{2}}\left\{e\left(V-V_{12}\right)+k_{\mathrm{B}} T_{12}\left[2 \ln \left(1+e^{\left.c\left(V_{1}-V\right) / /_{\mathrm{B}} T_{1}\right)}\right)-1\right]\right\} \\
& +2 \frac{S_{2} R_{2}^{2}}{R^{2}}\left\{e V_{12}+k_{\mathrm{B}} T_{12}\left[2 \ln \left(1+e^{-c / / /_{\mathrm{B}} T_{1}}\right)-1\right]\right\}
\end{aligned}
$$

The shot norse for two equal $\left(R_{1}=R_{2}\right)$ diffusive conductors,

$$
P=P_{\text {Polsson }} \frac{1}{\pi \sqrt{3}}[1+\ln 2+\ln \cosh (\pi / 2 \sqrt{3})] \simeq 038 P_{\text {Polsson }},
$$

1s slightly above the one-thi1d suppiession This shows that the cuirent becomes less correlated due to election-election scattering

(c) Inelastic scatteing This is the model of Ref [6] The distribution function of the intermediate 1eservoli is the Ferm1-Dirac distribution at the lattice temperature $T_{0}$, with an electiochemical potential $\mu_{12} \equiv E_{\mathrm{F}}+e V_{12}$, whe1e $V_{12}$ 1s given by $\mathrm{Eq}$ (5 10) This 1eservon absolbs energy, in contrast to cases (a) and (b) The zero-temperature shot-norse power follows fiom Eqs (5 5) and (56) [6]

$$
P=P_{\text {Poisson }} \frac{R_{1}^{3} S_{1}+R_{2}^{3} S_{2}}{R^{2}}
$$

For the diffusive case, w1th $R_{1}=R_{2}$, one has $P=\frac{1}{6} P_{\text {Porson }}$ The inelastic scatteing gives an additional suppiession [6]

For a double-ban1el system it is plausible to model the additional scattening by a single reservoli between the bat1ers In d diffusive conductor, however, these scatteing processes occul throughout the system It is therefore more realistic to divide the 
conductor into $M$ segments, connected by reservorrs. Eq. (5.5) becomes

$$
R^{2} P=\sum_{m=1}^{M} R_{m}^{2} P_{m}
$$

where the noise power $P_{m}$ of segment $m$ is calculated analogous to Eq. (5.6). We take the continuum limit $M \rightarrow \infty$. The electron distribution at position $x$ is denoted by $f(\varepsilon, x)$. At the ends of the conductor $f(\varepsilon, 0)=f_{\mathrm{L}}(\varepsilon)$ and $f(\varepsilon, L)=f_{\mathrm{R}}(\varepsilon)$, t.e. the electrons are Fermi-Dirac distributed at temperature $T_{0}$ and with electrochemical potential $\mu(0)=E_{\mathrm{F}}+e V$ and $\mu(L)=E_{\mathrm{F}}$, respectively. The value of $f(\varepsilon, x)$ inside the conductor depends on the type of scattering, (a), (b), or (c), and is determined below.

In the expression for $P_{m}$ only the first term of Eq. (5.6a) remanns. It follows from Eq. (5.17) that the noise power is given by

$$
P=\frac{4}{R^{2}} \int_{0}^{L} \mathrm{~d} x \frac{\rho(x)}{A} \int \mathrm{d} \varepsilon f(\varepsilon, x)[1-f(\varepsilon, x)],
$$

where $\rho(x)$ is the resistivity at position $x$. The total resistance is given by

$$
R=\frac{1}{A} \int_{0}^{L} \mathrm{~d} x \rho(x)
$$

For a constant resistivity $\rho$ we find from Eq. (5.18)

$$
P=\frac{4}{R L} \int_{0}^{L} \mathrm{~d} x \int \mathrm{d} \varepsilon f(\varepsilon, x)[1-f(\varepsilon, x)] .
$$

This formula has been derived by Nagaev from the Boltzmann-Langevin equation for 1sotropic 1mpurity scattering in the diffusive limit [7]. Our semiclassical calculation in the previous sections is worked out in teims of transmission piobabilities iather than in terms of the electron distribution function. However, one can casily convince oneself that in the diffusive limit and at zero temperature, Eqs (3.6) and (5.20) are equivalent. The present derivation shows that the quantum mechanical expression for the noise w1th phase-breaking rescrvoirs leads to the same result as the semiclassical approach. We evaluate Eq. (5 20) for the thiee types of scattering

(a) Quast-clastc scattering This calculation has previously bcen performed by $\mathrm{Na}$ gaev [7] and 1s similar to Section 3. Current conservation and the absence of inelastic scattering requires

$$
\frac{\partial^{2}}{\partial x^{2}} f(\varepsilon, x)=0
$$

The solution is

$$
f(\varepsilon, x)=\frac{L-x}{L} f(\varepsilon, 0)+\frac{x}{L} f(\varepsilon, L)
$$




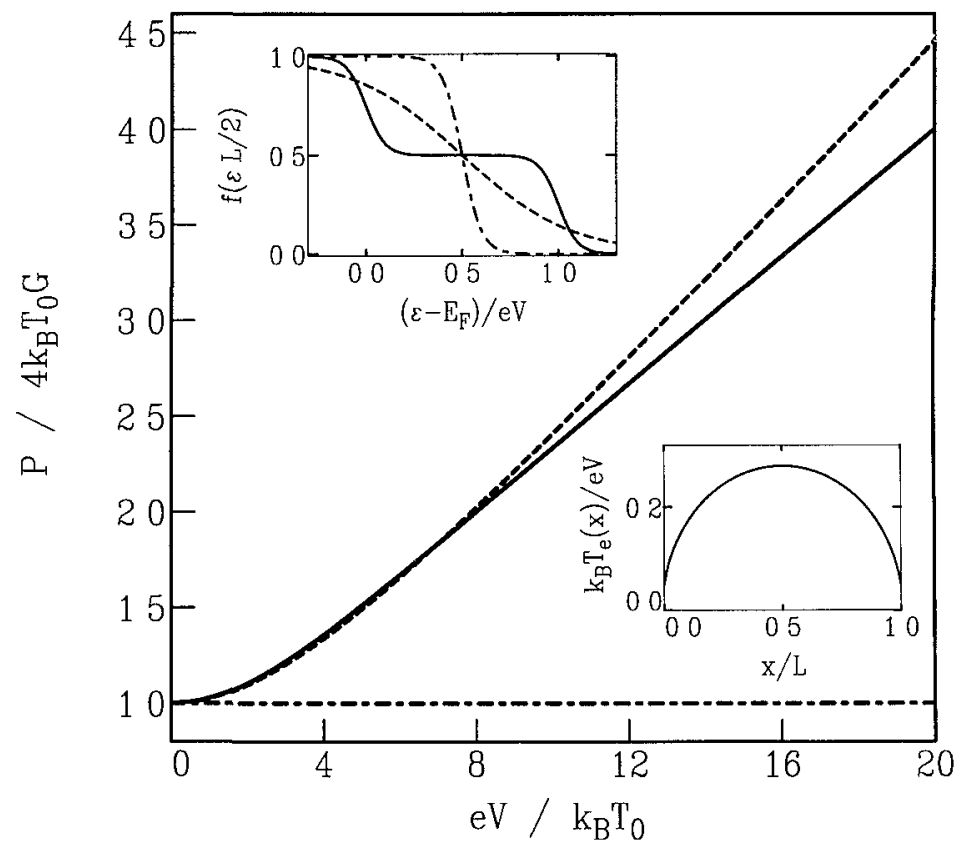

Fig 6 The nolse powe1 $P$ (divided by the Johnson-Nyqust noise $4 k_{\mathrm{B}} T_{0} G$ ) velsus applied voltage $V$ for a disordercd wite for model (a) of quasi elastic scattering (solid curve) (b) of election heating (dashed curve) (c) of inelastic scatteing (dash dotted curve) according to Eqs (523) (532) and (535) 1espectively The upper left inset gives the elcction distubution in the middle of the wite $f\left(\varepsilon \frac{1}{2} L\right)$ as a function of energy $\varepsilon$ for model (a) (b) and (c) The lower i ight inset shows the temperature $T_{\mathrm{c}}(x)$ as a function of the position $x$ for model (b) For both insets $k_{\mathrm{B}} T_{0}-\frac{1}{20} \mathrm{c}|V|$

The election distribution at $x=\frac{1}{2} L$ is plotted in the left inset of Fig 6 Substitution of Eq (5 22) 1nto Eq (5 20) yields [7]

$$
P=\frac{2}{3 R}\left[4 k_{\mathrm{B}} T_{0}+e V \operatorname{coth}\left(e V / 2 k_{\mathrm{B}} T_{0}\right)\right]
$$

At zero temperature the shot no1se is one-thi1d of the Poisson norse The temperature dependence of $P$ is given in Fig 6

(b) Electron heating This calculation is due to Martinis and Devoret [49] Simıla1 derivations on the basis of the Boltzmann-Langevin equation have been given by Nagaev [42] and by Kozub and Rudin [43] The election distibution function is a Ferm1-Dirac distubution at an elevated temperature $T_{\mathrm{e}}(r)$,

$$
f(\varepsilon, x)=\left\{1+\exp \left[\frac{\varepsilon-\mu(x)}{k_{\mathrm{B}} T_{\mathrm{c}}(x)}\right]\right\}^{\prime}
$$

The current density $f(\lambda)$ at $x$ is

$$
f(x)=-e D \mathcal{D}\left(E_{\mathrm{F}}\right) \frac{\partial}{\partial x} \int \mathrm{d} \varepsilon f(\varepsilon, x)
$$


where $D$ is the diffusion constant and $\mathcal{D}$ is the density of states We neglect the energy dependence of $D$ and $\mathcal{D}$ The resistivity $\rho$ is given by the Einstein relation, $\rho^{-1}=e^{2} D \mathcal{D}\left(E_{\mathrm{F}}\right)$ Current conservation yields

$$
\frac{\partial j(x)}{\partial x}=0 \text {, }
$$

which implies for the electrochemical potential

$$
\mu(x)=E_{\mathrm{F}}+\frac{L-x}{L} e V
$$

The eneigy-current density $J_{\varepsilon}(x)$ is determined accoiding to

$$
\begin{aligned}
& J_{\varepsilon}(x)=-D \mathcal{D}\left(E_{\mathrm{F}}\right) \frac{\partial}{\partial x} \int \mathrm{d} \varepsilon \varepsilon f(\varepsilon, x)=\mu(x) J(x) / e+J_{Q}(x), \\
& J_{Q}(x) \equiv-\kappa(x) \frac{\partial T_{\mathrm{c}}(x)}{\partial x}
\end{aligned}
$$

The heat-current density $J_{Q}(x)$ equals the temperature gradient times the heat conductivity $\kappa(x)=T_{\mathrm{c}}(x) \mathcal{L}_{0} / \rho$ Because of encigy conservation the divergence of the energycun ent density must be zero,

$$
\frac{\partial J_{\varepsilon}(x)}{\partial x}=0
$$

Combining Eqs (5 28) and (5 29), we obtan the following differential equation for the temperature

$$
\frac{\partial^{2}}{\partial x^{2}}\left[T_{c}(x)^{2}\right]=-\frac{2}{\mathcal{L}_{0}}\left(\frac{V}{L}\right)^{2}
$$

Taking into account the bounda1y conditions, the solution is

$$
T_{\mathrm{c}}(x)=\sqrt{T_{0}^{2}+(x / L)[1-(x / L)] V^{2} / \mathcal{L}_{0}}
$$

In the middle of the wire the electron temperature takes its maximum value For zeio lattice temperatule $\left(T_{0}=0\right)$ one has $k_{\mathrm{B}} T_{\mathrm{c}}\left(\frac{1}{2} L\right)=(\sqrt{3} / 2 \pi) e|V| \simeq 028 e|V|$ The election distribution at $x=\frac{1}{2} L$ is depicted in the left inset and the election temperature profile (5 31) 1s plotted in the 11ght inset of Fig 6

Eqs (5 20), (5 24), and (5 31) yreld for the norse power the result

$$
\begin{aligned}
P & =\frac{4}{R L} \int_{0}^{L} \mathrm{~d} x k_{\mathrm{B}} T_{\mathrm{c}}(x) \\
& =\frac{2 k_{\mathrm{B}} T_{0}}{R}+2 e I\left[\frac{2 \pi}{\sqrt{3}}\left(\frac{k_{\mathrm{B}} T_{0}}{e V}\right)^{2}+\frac{\sqrt{3}}{2 \pi}\right] \arctan \left(\frac{\sqrt{3}}{2 \pi} \frac{e V}{k_{\mathrm{B}} T_{0}}\right)
\end{aligned}
$$

Eq (5 32) is plotted in Fig 6 For the lumit $e V \gg k_{\mathrm{B}} T_{0}$ one finds [50]

$$
P=\frac{1}{4} \sqrt{3} P_{\text {Polsson }} \simeq 043 P_{\text {Polsson }}
$$


Due to the electron-electron scatteing the shot noise is increased The exchange of energies among the electrons makes the current less correlated The suppression factor of $\frac{1}{4} \sqrt{3}$ is close to the value observed in an experiment on silver wires by Steinbach et al [50]

(c) Inelastic scattering The electron distribution function is given by

$$
f(\varepsilon, x)=\left\{1+\exp \left[\frac{\varepsilon-\mu(x)}{k_{\mathrm{B}} T_{0}}\right]\right\}^{-1}
$$

with $\mu(x)$ according to Eq (5 27) For the noise power we obtain from Eqs (5 20) and (5 34)

$$
P=\frac{4 k_{\mathrm{B}} T_{0}}{R},
$$

which is equal to the Johnson-Nyquist noise for arbitrary $V$ (see Fig 6) The shot noise is thus completely suppressed by the inelastic scatteing $[6,13,42,43,51,52]$

These calculations assume a constant cross-section and resistivity of the conductor One might wonder, whether vatiations in cross-section and iesistivity, which will certainly appear in experiments, change the one-thind suppiession for the case of elastic scattering and the $\frac{1}{4} \sqrt{3}$ suppression for the case of electron-heating In Appendix D, it is demonstiated how this can be calculated on the basis of Eq (5 18) It is found that the results [Eqs (5 23), (5 32), and (5 35)] are independent of smooth variations in cross-section and 1esistivity We thus conclude, that both the one-thrrd suppiession as well as the $\frac{1}{4} \sqrt{3}$ suppiession aie in principle observable in any diffusive conductor

\section{Conclusions and discussion}

We have derived a general formula for the shot norse within the framework of the semiclassical Boltzmann-Langevin equation We have applied this to the case of a disordered conductor, wherc we have calculated how the shot norse crosses over fiom complete suppiession in the ballistic limit to one-third of the Poisson noise in the diffusive limit Furthermore, we have applied our formula to the shot norse in a conductor consisting of a sequence of tunnel barries Finally, we have considered a disordered conductor in senes with a tunnel ban1er For all these systems, we have obtamed a sub-Poissonian shot-notse powe1, in complete agieement with quantum mechanical calculations in the literature This establishes that phase coherence is not required for the occurrence of suppressed shot noise in mesoscopic conductors Moreove1, it has been shown that for diffusive conductors the one-thid suppiession occuis quite generally This phenomenon depends nether on the dimensionality of the conducto1, nor on the microscopic details of the scattermg potential

We have modeled quasi-elastic scatteing (which bieaks phase coherence), election heatıng (due to election-election scatteing), and inelastic scatteing (due to, e g, election-phonon scatteing) by putting change-conserving reservous between phasecoherent segments of the conductor If the scattering occurs throughout the whole 
length of the conductor, we end up with the same formula for the norse as can be obtained directly from the Boltzmann-Langevin approach $[42,43]$ In the case of election heating, the shot noise is $\frac{1}{4} \sqrt{3}$ of the Poisson noise, which is slightly above $\frac{1}{3} P_{\text {Poisson }}$ for the fully elastic case The experiments of Refs [11,50] are likely in this electionheating regime We have demonstrated that both the one-third suppression and the $\frac{1}{4} \sqrt{3}$ suppression are insensitive to the geometry of the conductor, as long as the transport is in the diffusive regime For future wolk, it might be worthwhile to take the effects of electron heating and inelastic scattering into account through the scatleung terms in the Boltzmann-Langevin equation, as has been done in Refs $[42,43]$, in order to calculate the crossover between the different regimes

In both the quantum mechanical and semiclassical theories the elections are treated as noninteracting particles Some aspects of the electron-electron interaction are taken into account by the conditions on the reservorrs in Section 5, where fluctuations in the electrostatic potential enforce charge-neutrality We have shown that these fluctuations suppress the noise only in the presence of inelastic scattering Coulomb repulsion is known to have a strong effect on the noise in confined geometries with a small capac1tance $[39,53]$ This is relevant for the double-barrier case tieated in Section 4 Theor res which take the Coulomb blockade into account $[39,53]$ predict a shot-noise suppiession which is periodic in the applied voltage This effect has recently been observed for a nanoparticle between a surface and the tip of a scanning tunneling micioscope [54] In open conductors we would expect these interaction effects to be less important [55]

\section{Acknowledgements}

We thank MH Devoret and R Landauer for valuable discussions This research has been supported by the "Nederlandse organısatı voor Wetenschappelijk Onderzoek" (NWO) and by the "Stichting voor Fundamenteel Onderzoek der Matel1e" (FOM)

\section{Appendix A. Thermal noise}

In this appendix it is shown how theimal fluctuations can be incorporated in the theory These are ignored in Sections 3 and 4 where zeio tempeiature is considered At nonzero temperatures we need to take into account the time-dependent fluctuations in the occupation of the states in the reservors The formal solution of the BoltzmannLangevin equation (24) can bc written as

$$
\begin{aligned}
\delta f(\mathbf{r}, \mathbf{k}, t)= & \int_{-\infty}^{t} \mathrm{~d} t^{\prime} \int_{\mathcal{V}} \mathrm{d} \mathbf{r}^{\prime} \int \mathrm{d} \mathbf{k}^{\prime} \mathcal{G}\left(\mathbf{r}, \mathbf{k}, \mathbf{r}^{\prime}, \mathbf{k}^{\prime}, t-t^{\prime}\right) J\left(\mathbf{r}^{\prime}, \mathbf{k}^{\prime}, t^{\prime}\right) \\
& +\int_{\infty}^{t} \mathrm{~d} t^{\prime} \int_{S_{L}} \mathrm{~d} \mathbf{y}^{\prime} \int_{k>0} \mathrm{~d} \mathbf{k}^{\prime} v_{x} \mathcal{G}\left(\mathbf{r}, \mathbf{k}, \mathbf{r}^{\prime}, \mathbf{k}^{\prime}, t-t^{\prime}\right) \delta f\left(\mathbf{r}^{\prime}, \mathbf{k}^{\prime}, t^{\prime}\right)
\end{aligned}
$$




$$
+\int_{\infty}^{t} \mathrm{~d} t^{\prime} \int_{S_{R}} \mathrm{~d} \mathbf{y}^{\prime} \int_{l<0} \mathrm{~d} \mathbf{k}^{\prime}\left|v_{x}\right| \mathcal{G}\left(\mathbf{r}, \mathbf{k}, \mathbf{r}^{\prime}, \mathbf{k}^{\prime}, t-t^{\prime}\right) \delta f\left(\mathbf{r}^{\prime}, \mathbf{k}^{\prime}, t^{\prime}\right)
$$

where $\mathcal{V}$ denotes the scattering region of the conductor The second and this term describe the time-dependent fluctuations of states originating fiom the reservoli which are ignoied in Eq (2 17) The conelation function of the incoming fluctuations which have not yet reached the scattering 1egion $\left[1 \mathrm{e}\right.$ for the left lead $x, x^{\prime} \leqslant x_{\mathrm{L}}, k_{\mathrm{r}}, k_{\mathrm{r}}^{\prime}>0$ and for the 1ight lead $x, x^{\prime} \geqslant x_{\mathrm{R}}, k_{\mathrm{u}}, k_{x}^{\prime}<0$ ] follows fiom Eqs (2 8) and (29)

$$
\begin{aligned}
\left\langle\delta f(\mathbf{r}, \mathbf{k}, t) \delta f\left(\mathbf{r}^{\prime}, \mathbf{k}^{\prime}, t^{\prime}\right)\right\rangle= & (2 \pi)^{d} \delta\left[\mathbf{r}-\mathbf{r}^{\prime}-\mathbf{v}\left(t-t^{\prime}\right)\right] \delta\left(\mathbf{k}-\mathbf{k}^{\prime}\right) \\
& \times f_{\mathrm{LR}}(\varepsilon)\left[1-f_{\mathrm{LR}}(\varepsilon)\right]
\end{aligned}
$$

The derivation of the noise power proceeds similar to the deilvation of $\mathrm{Eq}$ (2 17) Substitution of Eq (A 1) into Eqs (2 11) and (2 12) and using both the conclation functions (2 5) and (A 2), yields

$$
\begin{aligned}
P= & \frac{2 e^{2}}{(2 \pi)^{d}}\left(\int_{\nu} \mathrm{d} \mathbf{r} \int \mathrm{d} \mathbf{k} \int \mathrm{d} \mathbf{k}^{\prime} T(\mathbf{r}, \mathbf{k}) T\left(\mathbf{r}, \mathbf{k}^{\prime}\right) J\left(\mathbf{1}, \mathbf{k}, \mathbf{k}^{\prime}\right)\right. \\
& +\int_{S_{l}} \mathrm{~d} \mathbf{y} \int_{k>0} \mathrm{~d} \mathbf{k} v_{\imath} T(\mathbf{r}, \mathbf{k})^{2} f_{\mathrm{L}}(\varepsilon)\left[1-f_{\mathrm{L}}(\varepsilon)\right] \\
& \left.+\int_{S_{I}} \mathrm{~d} \mathbf{y} \int_{l<0} \mathrm{~d} \mathbf{k}\left|v_{1}\right|[1-T(\mathbf{r}, \mathbf{k})]^{2} f_{\mathrm{R}}(\varepsilon)\left[1-f_{\mathrm{R}}(\varepsilon)\right]\right)
\end{aligned}
$$

Let us apply Eq (A 3) to the case of mpurty scattenng, tieated in Section 3 for zero temperature By changing variables accotding to $\mathrm{Eq}$ ( 31 ) and by substitution of Eqs (2 6) and (3 3), we obtain

$$
\begin{aligned}
P= & 2 e^{2} A \int_{0}^{l} \mathrm{~d} \imath \int \mathrm{d} \varepsilon \mathcal{D}(\varepsilon) \int \frac{\mathrm{d} \hat{\mathbf{n}}}{s_{d}} \int \frac{\mathrm{d} \hat{\mathbf{n}}^{\prime}}{s_{d}} W_{\mathbf{n n}}\left[T(\imath, \hat{\mathbf{n}})-T\left(\lambda, \hat{\mathbf{n}}^{\prime}\right)\right]^{2} \\
& \times\left\{f_{\mathrm{L}}(\varepsilon)\left[1-f_{\mathrm{L}}(\varepsilon)\right][1-T(x,-\hat{\mathbf{n}})]+f_{\mathrm{R}}(\varepsilon)\left[1-f_{\mathrm{R}}(\varepsilon)\right] T(\lambda,-\hat{\mathbf{n}})\right. \\
& \left.+\left[f_{\mathrm{L}}(\varepsilon)-f_{\mathrm{R}}(\varepsilon)\right]^{2} T(x,-\hat{\mathbf{n}})\left[1-T\left(x,-\hat{\mathbf{n}}^{\prime}\right)\right]\right\} \\
& +2 e^{2} A \int \mathrm{d} \varepsilon \mathcal{D}(\varepsilon) f_{\mathrm{L}}(\varepsilon)\left[1-f_{\mathrm{L}}(\varepsilon)\right] \int \frac{\mathrm{d} \hat{\mathbf{n}}}{s_{l l}} v n_{1} T^{2}(0, \hat{\mathbf{n}}) \\
& -2 e^{2} A \int \mathrm{d} \varepsilon \mathcal{D}(\varepsilon) f_{\mathrm{R}}(\varepsilon)\left[1-f_{\mathrm{R}}(\varepsilon)\right] \int \frac{\mathrm{d} \hat{\mathbf{n}}}{s_{d}} v n_{1}[1-T(L, \hat{\mathbf{n}})]^{2},
\end{aligned}
$$

where we have used Eq (3 2) and $W_{\mathrm{nn}}=W_{\mathbf{n} \text { n }} \mathrm{Eq}$ (A 4) can be simplified by means of the relations

$$
\int_{0}^{l} \mathrm{~d} \downarrow \int \frac{\mathrm{d} \hat{\mathbf{n}}}{s_{d}} \int \frac{\mathrm{d} \hat{\mathbf{n}}^{\prime}}{s_{d l}} W_{\mathbf{n n}}\left[T(x, \hat{\mathbf{n}})-T\left(x, \hat{\mathbf{n}}^{\prime}\right)\right]^{2}=v_{\mathrm{r}} \int \frac{\mathrm{d} \hat{\mathbf{n}}}{s_{d l}} n_{1}\left[T^{2}(L, \hat{\mathbf{n}})-T^{2}(0, \hat{\mathbf{n}})\right],
$$




$$
\begin{aligned}
& \int_{0}^{L} \mathrm{~d} x \int \frac{\mathrm{d} \hat{\mathbf{n}}}{s_{d}} \int \frac{\mathrm{d} \hat{\mathbf{n}}^{\prime}}{s_{d}} W_{\hat{\mathbf{n}} \hat{\mathbf{n}}^{\prime}}\left[T(x, \hat{\mathbf{n}})-T\left(x, \hat{\mathbf{n}}^{\prime}\right)\right]^{2} T(x,-\hat{\mathbf{n}}) \\
& \quad=v_{\mathrm{F}} \int \frac{\mathrm{d} \hat{\mathbf{n}}}{s_{d}} n_{x}\left[T^{2}(L, \hat{\mathbf{n}}) T(L,-\hat{\mathbf{n}})-T^{2}(0, \hat{\mathbf{n}}) T(0,-\hat{\mathbf{n}})\right],
\end{aligned}
$$

which can be derived fiom Eq. (3.2). For the distribution function we apply the identity $f_{0}\left(1-f_{0}\right)=-k_{\mathrm{B}} T_{0} \partial f_{0} / \partial \varepsilon$ and define

$$
F\left(V, T_{0}\right) \equiv \int \mathrm{d} \varepsilon\left[f_{\mathrm{L}}(\varepsilon)-f_{\mathrm{R}}(\varepsilon)\right]^{2}=e|V| \operatorname{coth}\left(\frac{e|V|}{2 k_{\mathrm{B}} T_{0}}\right)-2 k_{\mathrm{B}} T_{0} .
$$

Collecting results, we find for the noise power the expression

$$
\begin{aligned}
P= & \frac{2 F\left(V, T_{0}\right) G_{0} N}{v_{\mathrm{F}} s_{d} v_{d-1}} \int_{0}^{L} \mathrm{~d} x \int \mathrm{d} \hat{\mathbf{n}} \int \mathrm{d} \hat{\mathbf{n}}^{\prime} W_{\hat{\mathbf{n}} \mathbf{n}^{\prime}}\left[T(x, \hat{\mathbf{n}})-T\left(x, \hat{\mathbf{n}}^{\prime}\right)\right]^{2} \\
& \times T(x,-\hat{\mathbf{n}})\left[1-T\left(x,-\hat{\mathbf{n}}^{\prime}\right)\right]+4 k_{\mathrm{B}} T_{0} G_{0} N \int \frac{\mathrm{d} \hat{\mathbf{n}}}{v_{d-1}} n_{\lambda} T(L, \hat{\mathbf{n}})
\end{aligned}
$$

At zero voltage, Eqs. (3.4) and (A.8) reduce to the Johnson-Nyquist noise $P=4 k_{\mathrm{B}} T_{0} G$. At zero temperature, Eq. (A.8) reduces to Eq. (3.6) Applying Eq. (A.8) to impurity scatterng for the case $d=1$ of Section 3, we obtain

$$
P=\frac{2 G}{3}\left\{e V \operatorname{coth}\left(\frac{e V}{2 k_{\mathrm{B}} T_{0}}\right)\left[1-\frac{1}{(1+L / \tilde{\varphi})^{3}}\right]+2 k_{\mathrm{B}} T_{0}\left[2+\frac{1}{(1+L / \tilde{\ell})^{3}}\right]\right\} .
$$

The voltage dependence of the noise is plotted in Fig. 7 for various values of $L / \tilde{\ell}$. The result for the diffusive limit is equal to Eq. (5.23). Also depicted is the classical result for a single high tunnel barrier $(\Gamma \ll 1)$,

$$
P=2 e|I| \operatorname{coth}\left(\frac{e V}{2 k_{\mathrm{B}} T_{0}}\right)
$$

which can be derived within our theory by combining the results of Section 4 with the analysis of this appendix.

\section{Appendix B. Noise at arbitrary cross-section}

Let us verify that the noise power does not depend on the location $x$ of the crosssection at which the current is cvaluated The fluctuating current through a cross-section $S_{x}$ at coordinate $x$ is defined by

$$
\delta I(t, x) \equiv \frac{e}{(2 \pi)^{d}} \int_{S_{1}} \mathrm{~d} \mathbf{y} \int \mathrm{d} \mathbf{k} v_{\varkappa} \delta f(\mathbf{r}, \mathbf{k}, t)
$$




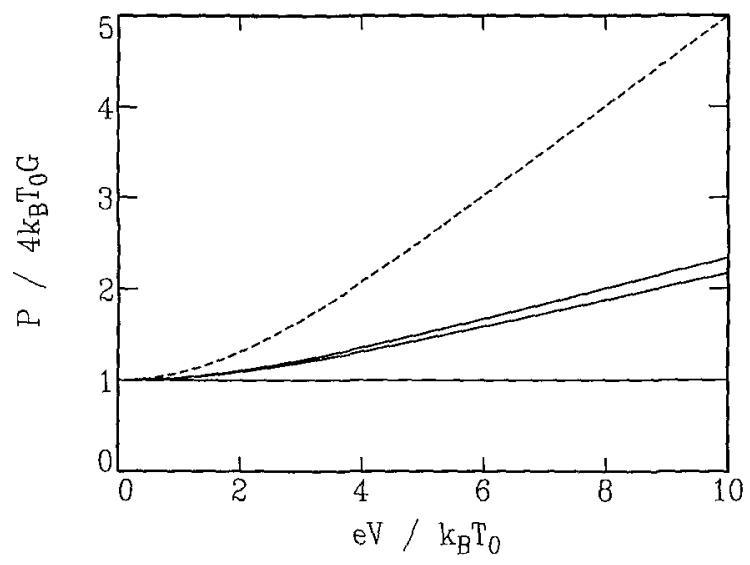

Fig 7 The noise powe1 $P$ (divided by the Johnson-Nyquist noise $4 k_{\mathrm{B}} T_{0} G$ ) veisus applied voltage $V$ for a disordeied wite (bottom to top) in the ballistic limit $L / \tilde{l} \rightarrow 0$, the intermediate regime $L / \tilde{f}=1$, and in the diffusive limit $L / \tilde{t} \rightarrow \infty$, as given by Eq (A 9) The dashed line is the noise in a tunnel barties, according to $\mathrm{Eq}$ (A 10)

and leads to

$$
P\left(x, x^{\prime}\right) \equiv 2 \int_{-\infty}^{\infty} \mathrm{d} t\left\langle\delta I(t, x) \delta I\left(0, x^{\prime}\right)\right\rangle
$$

We use the following relation

$$
\int_{0}^{\infty} \mathrm{d} t \int_{S_{\mathfrak{\imath}}} \mathrm{d} \mathbf{y} \int \mathrm{d} \mathbf{k} v_{\lambda} \mathcal{G}\left(\mathbf{r}, \mathbf{k} ; \mathbf{r}_{0}, \mathbf{k}_{0} ; t\right)=T\left(\mathbf{r}_{0}, \mathbf{k}_{0}\right)-\Theta\left(x_{0}-x\right),
$$

which follows from Eqs. (2.14) and (2.15). Here, $\Theta(x)$ is the unit-step function. Evaluating Eq. (B.2) along the lines of Section 2, we find

$$
\begin{aligned}
P\left(x, x^{\prime}\right)= & \frac{2 e^{2}}{(2 \pi)^{d}} \int \mathrm{d} \mathbf{r}_{0} \int \mathrm{d} \mathbf{k}_{0} \int \mathrm{d} \mathbf{k}_{0}^{\prime} J\left(\mathbf{r}_{0}, \mathbf{k}_{0}, \mathbf{k}_{0}^{\prime}\right) \\
& \times\left[T\left(\mathbf{r}_{0}, \mathbf{k}_{0}\right)-\Theta\left(x_{0}-x\right)\right]\left[T\left(\mathbf{r}_{0}, \mathbf{k}_{0}^{\prime}\right)-\Theta\left(x_{0}-x^{\prime}\right)\right] .
\end{aligned}
$$

We use the fact that the integral over $\mathbf{k}$ or over $\mathbf{k}^{\prime}$ of $J\left(\mathbf{r}, \mathbf{k}, \mathbf{k}^{\prime}\right)$ vanishes, Eq. (2.7), and find that $P\left(x, x^{\prime}\right)$ is independent of $x, x^{\prime}$.

\section{Appendix C. Nonisotropic scattering}

We w1sh to demonstrate that the occurrence of one-third suppressed shot noise in the diffusive regime is independent of the angle-dependence of the scattering rate. We write $W_{\hat{\mathbf{n}} \hat{\mathbf{n}}^{\prime}}==w\left(\hat{\mathbf{n}} \cdot \hat{\mathbf{n}}^{\prime}\right) v_{\mathrm{F}}$, with arbitiary $w$. In the diffusive limit, the transmission probability 1s given by

$$
T(x, \hat{\mathbf{n}})=T(x)+t\left(n_{\imath}\right),
$$




$$
\begin{aligned}
& \int_{0}^{I} \mathrm{~d} x \int \frac{\mathrm{d} \hat{\mathbf{n}}}{s_{d}} \int \frac{\mathrm{d} \hat{\mathbf{n}}^{\prime}}{s_{d}} W_{\mathbf{n n}^{\prime}}\left[T(x, \hat{\mathbf{n}})-T\left(x, \hat{\mathbf{n}}^{\prime}\right)\right]^{2} T(x,-\hat{\mathbf{n}}) \\
& \quad=v_{\mathrm{F}} \int \frac{\mathrm{d} \hat{\mathbf{n}}}{s_{d}} n_{\lambda}\left[T^{2}(L, \hat{\mathbf{n}}) T(L,-\hat{\mathbf{n}})-T^{2}(0, \hat{\mathbf{n}}) T(0,-\hat{\mathbf{n}})\right],
\end{aligned}
$$

which can be derived fiom Eq (3 2) For the distribution function we apply the identity $f_{0}\left(1-f_{0}\right)=-k_{\mathrm{B}} T_{0} \partial f_{0} / \partial \varepsilon$ and define

$$
F\left(V, T_{0}\right) \equiv \int \mathrm{d} \varepsilon\left[f_{\mathrm{L}}(\varepsilon)-f_{\mathrm{R}}(\varepsilon)\right]^{2}=e|V| \operatorname{coth}\left(\frac{e|V|}{2 k_{\mathrm{B}} T_{0}}\right)-2 k_{\mathrm{B}} T_{0}
$$

Collecting 1esults, we find for the norse power the expression

$$
\begin{aligned}
P= & \frac{2 F\left(V, T_{0}\right) G_{0} N}{v_{\mathrm{F}} s_{d} v_{d-1}} \int_{0}^{L} \mathrm{~d} x \int \mathrm{d} \hat{\mathbf{n}} \int \mathrm{d} \hat{\mathbf{n}}^{\prime} W_{\mathbf{n n}^{\prime}}\left[T(x, \hat{\mathbf{n}})-T\left(x, \hat{\mathbf{n}}^{\prime}\right)\right]^{2} \\
& \times T(x,-\hat{\mathbf{n}})\left[1-T\left(x,-\hat{\mathbf{n}}^{\prime}\right)\right]+4 k_{\mathrm{B}} T_{0} G_{0} N \int \frac{\mathrm{d} \hat{\mathbf{n}}}{v_{d}{ }_{1}} n_{\mathrm{r}} T(L, \hat{\mathbf{n}})
\end{aligned}
$$

At zero voltage, Eqs (3 4) and (A 8) reduce to the Johnson-Nyquist noise $P=4 k_{\mathrm{B}} T_{0} G$ At zeio tempeiature, Eq (A 8) reduces to Eq (36) Applying Eq (A 8) to 1mpuity scattering for the case $d=1$ of Section 3, we obtan

$$
P=\frac{2 G}{3}\left\{e V \operatorname{coth}\left(\frac{e V}{2 k_{\mathrm{B}} T_{0}}\right)\left[1-\frac{1}{(1+L / \tilde{\ell})^{3}}\right]+2 k_{\mathrm{B}} T_{0}\left[2+\frac{1}{(1+L / \tilde{\rho})^{3}}\right]\right\}
$$

The voltage dependence of the noise is plotted in Fig 7 for various values of $L / \tilde{\ell}$ The result for the diffusive limit is equal to $\mathrm{Eq}$ (523) Also depicted is the classical result for a single high tunnel bairer $(\Gamma \ll 1)$,

$$
P=2 e|I| \operatorname{coth}\left(\frac{e V}{2 k_{\mathrm{B}} T_{0}}\right),
$$

which can be delived within our theory by combining the results of Section 4 with the andysis of this appendix

\section{Appendix B. Noise at arbitrary cross-section}

Let us verify that the noise power does not depend on the location $x$ of the c1osssection at which the curient is evaluated The fluctuating curient thiough a cioss-section $S_{\mathrm{r}}$ at cooldinate $x$ is defined by

$$
\delta I(t, x) \equiv \frac{e}{(2 \pi)^{\prime \prime}} \int_{S} \mathrm{~d} \mathbf{y} \int \mathrm{d} \mathbf{k} v_{\imath} \partial f(\mathbf{r}, \mathbf{k}, i)
$$




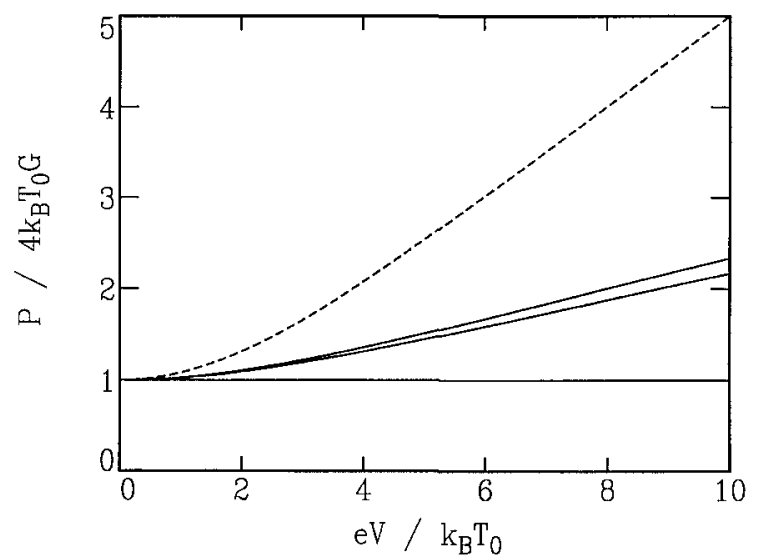

Fig 7 The noise power $P$ (divided by the Johnson Nyquist norse $4 k_{\mathrm{B}} T_{0} G$ ) versus applied voltage $V$ for a disoldered wire (bottom to top) in the ballistic limit $L / \tilde{\ell} \rightarrow 0$, the intermediate regime $L / \tilde{f}=1$, and in the diffusive limit $L / \tilde{l} \rightarrow \infty$, as given by Eq (A 9) The dashed line is the noise in a tunnel banter, according to $\mathrm{Eq}(\mathrm{A} 10)$

and leads to

$$
P\left(x, x^{\prime}\right) \equiv 2 \int_{-\infty}^{\infty} \mathrm{d} t\left\langle\delta I(t, x) \delta I\left(0, x^{\prime}\right)\right\rangle
$$

We use the following relation

$$
\int_{0}^{\infty} \mathrm{d} t \int_{S} \mathrm{~d} \mathbf{y} \int \mathrm{d} \mathbf{k} v_{x} \mathcal{G}\left(\mathbf{r}, \mathbf{k}, \mathbf{r}_{0}, \mathbf{k}_{0}, t\right)=T\left(\mathbf{r}_{0}, \mathbf{k}_{0}\right)-\Theta\left(x_{0}-x\right),
$$

which follows fiom Eqs (2 14) and (2 15) Here, $\Theta(x)$ is the unit-step function Evaluatıng Eq (B 2) along the lines of Section 2, we find

$$
\begin{aligned}
P\left(x, x^{\prime}\right)= & \frac{2 e^{2}}{(2 \pi)^{d}} \int \mathrm{d} \mathbf{r}_{0} \int \mathrm{d} \mathbf{k}_{0} \int \mathrm{d} \mathbf{k}_{0}^{\prime} J\left(\mathbf{r}_{0}, \mathbf{k}_{0}, \mathbf{k}_{0}^{\prime}\right) \\
& \times\left[T\left(\mathbf{r}_{0}, \mathbf{k}_{0}\right)-\Theta\left(x_{0}-x\right)\right]\left[T\left(\mathbf{r}_{0}, \mathbf{k}_{0}^{\prime}\right)-\Theta\left(x_{0}-x^{\prime}\right)\right]
\end{aligned}
$$

We use the fact that the integial over $\mathbf{k}$ or over $\mathbf{k}^{\prime}$ of $J\left(\mathbf{r}, \mathbf{k}, \mathbf{k}^{\prime}\right)$ vanıshes, Eq (27), and find that $P\left(x, x^{\prime}\right)$ is independent of $x, x^{\prime}$

\section{Appendix C. Nonisotropic scattering}

We wish to demonstiate that the occurience of one-thild suppiessed shot noise in the diffusive 1egime is independent of the angle-dependence of the scatteing rate We wite $W_{\mathbf{n i}^{\prime}}=w\left(\hat{\mathbf{n}} \hat{\mathbf{n}}^{\prime}\right) v_{\mathrm{F}}$, with arbitiary $w$ In the diffusive limit, the transmission piobability is given by

$$
T(x, \hat{\mathbf{n}})=T(x)+t\left(n_{\imath}\right),
$$


where $T(x)=x / L$ and $t\left(n_{x}\right)$ of order $\tilde{\ell} / L$, with $\int \mathrm{d} \hat{\mathbf{n}} t\left(n_{x}\right)=0$ The conductance 1 given by the Drude result, Eq (38), where the normalized mean free path $\tilde{\ell}$ can be derved as follows Upon integration of $\mathrm{Eq}$ (32a) over $\mathrm{d} \hat{\mathbf{n}} n_{x}$ and substitution of Eq (C 1), one obtains

$$
\int \frac{\mathrm{d} \hat{\mathbf{n}}}{s_{d}} n_{\lambda}^{2} \frac{\mathrm{d} T(x)}{d x}=\int \frac{\mathrm{d} \hat{\mathbf{n}}}{s_{d}} \int \frac{\mathrm{d} \hat{\mathbf{n}}^{\prime}}{s_{d}} n_{\lambda} w\left(\hat{\mathbf{n}} \quad \hat{\mathbf{n}}^{\prime}\right)\left[t\left(n_{x}\right)-t\left(n_{r}^{\prime}\right)\right]
$$

Comparison with Eq (3 4) yields

$$
\tilde{\ell}=\frac{v_{d}}{v_{d} 1}\left[\int \frac{\mathrm{d} \hat{\mathbf{n}}}{s_{d}} w\left(n_{x}\right)\left(1-n_{\imath}\right)\right]^{-1}
$$

From $\mathrm{Eq} \mathrm{(32a)} \mathrm{it} \mathrm{also} \mathrm{follows} \mathrm{that}$

$$
\begin{aligned}
& \int \frac{\mathrm{d} \hat{\mathbf{n}}}{s_{d}} \int \frac{\mathrm{d} \hat{\mathbf{n}}^{\prime}}{s_{d}} W_{\mathbf{n n}}\left[T(x, \hat{\mathbf{n}})-T\left(x, \hat{\mathbf{n}}^{\prime}\right)\right]^{2} \\
& =v_{\mathrm{F}} \frac{\partial}{\partial x} \int \frac{\mathrm{d} \hat{\mathbf{n}}}{s_{d}} n_{\lambda} T^{2}(x, \hat{\mathbf{n}}) \\
& =\frac{2 G v_{\Gamma} v_{d-1}}{G_{0} N s_{d}} \frac{\mathrm{d} T(x)}{\mathrm{d} x}
\end{aligned}
$$

where we have used Eqs (3 4) and (C 1) By substitution of Eq (C 4) into Eq (3 6) and neglecting terms of order $\tilde{\ell} / L$, we find

$$
P=2 P_{\text {Poisson }} \int_{0}^{L} \mathrm{~d} x T(x)[1-T(x)] \frac{\mathrm{d} T(x)}{\mathrm{d} x}=\frac{1}{3} P_{\text {Poisson }},
$$

1ndependent of $w$

\section{Appendix D. The effect of variations in cross-section and resistivity}

In Section 5, we have calculated the shot noise in a diffusive conductor for several types of scattering It has been assumed that both the area of the cross-section $A$ and the resistivity $\rho$ are constant along the conductor Below, we buefly describe how the calculations are modified by taking into account a non-constant, but smoothly varying area $A(x)$ and resistivity $\rho(x)$

Out starting point is $\mathrm{Eq}(518)$ It 1 s convenient to change valiables from $x$ to $\eta$, defined according to

$$
\eta \equiv \frac{1}{R} \int_{0}^{r} \mathrm{~d} x^{\prime} \frac{A\left(x^{\prime}\right)}{\rho\left(x^{\prime}\right)}
$$

In other words, $\eta$ is the ratio between the resistance of the conductor fiom 0 to $x$ and the total resistance $\mathrm{Eq}$ (5 18) thus becomes 


$$
P=\frac{4}{R} \int_{0}^{1} \mathrm{~d} \eta \int \mathrm{d} \varepsilon f(\varepsilon, \eta)[1-f(\varepsilon, \eta)] .
$$

It is now straightforward to repeat the calculation for the diffusive conductor in Section 5. It follows, that all the results [Eqs (5.23), (5 32), and (5.35)] remain unaltered Here, we will just 1llustrate how the calculation for the case of electron heating is done.

Starting from Eq (5.24) we find for the current at position $\eta$

$$
I(\eta)=-\frac{1}{e R} \frac{\partial}{\partial \eta} \mu(\eta)
$$

From current conservation $\{I(\eta)=I$ for all $\eta \in[0,1]\}$ it follows that the electrochemical potential is

$$
\mu(\eta)=E_{\mathrm{F}}+(1-\eta) e V
$$

The energy current is given by

$$
I_{\varepsilon}(\eta)=\frac{\mu(\eta) I}{e}-\frac{\mathcal{L}_{0}}{R} T_{\mathrm{e}}(\eta) \frac{\partial}{\partial \eta} T_{\mathrm{c}}(\eta) .
$$

Similar to the detivation in Section 5, we thus find

$$
T_{\mathrm{e}}(\eta)=\sqrt{T_{0}^{2}+\eta(1-\eta) V^{2} / \mathcal{L}_{0}}
$$

from which it follows that the noise is given by Eq. (5.32), as before.

\section{References}

[1] V A Khlus, Zh Eksp Teor Frz 93 (1987) 2179 [Sov Phys JETP 66 (1987) 1243]

[2] G B Lesovik, Pıs'ma Zh Eksp Teoı Fiz 49 (1989) 513 [JETP Lett 49 (1989) 592]

[3] B Yu1ke and GP Kochanski, Phys Rev B 41 (1990) 8184

[4] M Buttıkeı, Phys Rev Lett 65 (1990) 2901, Phys Rev B 46 (1992) 12485

[5] Th Martin and R Landauer, Phys Rev B 45 (1992) 1742

[6] C W J Beenakker and M Buttker, Phys Rev B 46 (1992) 1889

[7] K E Nagaev, Phys Lett A 169 (1992) 103

[8] M J M de Jong and C W J Beenakker, Phys Rev B 46 (1992) 13400

[9] Yu V Naza1ov, Phys Rev Lett 73 (1994) 134

[10] B L Altshuler, L S Levitov and A Yu Yakovets, Pis'ma Zh Eksp Teor Fiz 59 (1994) 821 [JETP Lett 59 (1994) 857]

[11] F Licfiınk, J I Dijkhuis, MJM de Jong, LW Molenkamp, and H van Houten, Phys Rev B 49 (1994) 14066

[12] O D Dolokhov, Solid State Commun 51 (1984) 381, Y Imry, Eu1ophys Lett 1 (1986) 249, J B Pendiy, A MacKinnon and P J Roberts, Pioc R Soc London Se1 A 437 (1992) 67

[13] A Shımızu and M Ueda, Phys Rev Lett 69 (1992) 1403

[14] R Landauer, piepıint

[15] M I M de Jong, Phys Rev B 49 (1994) 7778

[16] B B Kadomtsev, Zh Eksp Teor Fiz 32 (1957) 943 [Sov Phys JETP 5 (1957) 771]

[17] Sh M Kogan and A Ya Shul'man, Zh Eksp Teor Fiz 56 (1969) 862 [Sov Phys JETP 29 (1969) 467] 
[18] IO Kulık and A N Omel'yanchuk, F1z Nizk Temp 10 (1984) 305 [Sov J Low Temp Phys 10 (1984) 158]

[19] C W J Beenakker and H van Houten, Phys Rev B 43 (1991) 12066 The results presented in this paper are incorrect, because the equal-tıme correlation (Eq (6) of Ref [19]) is not generally valid

[20] M J M de Jong and C W J Beenakker, Phys Rev B 51 (1995) 16867

[21] L Y Chen and C S Ting, Phys Rev B 43 (1991) 4534

[22] J H Davies, P Hyldgaard, S Hershfield and J W Wilkins, Phys Rev B 46 (1992) 9620

[23] LS Levitov and GB Lesovik, PIs'ma Zh Eksp Teor Fiz 58, 225 (1993) [JETP Lett 5£ (1993) 230]

[24] H Lee, L S Levitov and A Yu Yakovets, Phys Rev B 51 (1995) 4079

[25] Y P L1, D C Tsu1, J J Hercmans, J A Simmons and G W Weımann, Appl Phys Lett 57 (1990) 774

[26] M Reznıkov, M Herblum, H Shtrikman and D Mahalu, Phys Rev Lett 76 (1995) 3340

[27] A Kumar, L Samınadayar, D C Glattlı, Y Jin and B Etıenne, preprint

[28] M JM de Jong and CW J Beenakkeı, in Coulomb and Interference Effects in Small Electiontc Stıuctures, eds D C Glattlı, M Sanquer and J Trân Thanh Vân (Edıtıons Fiontıeres, France, 1994)

[29] R Landauer, Ann N Y Acad Sc1 755 (1995) 417

[30] S V Gantsevich, V L Gurevich and R Katilus, Rivista Nuovo Cimento 2(5) (1979) 1

[31] O M Bulashenko and VA Kochelap, J Phys Condens Matter 5 (1993) L469

[32] C W J Beenakke1 and H van Houten, Phys Rev Lett 63 (1989) 1857

[33] Yu V Sharvin, Zh Eksp Teor Fiz 48 (1965) 984 [Sov Phys JETP 21 (1965) 655]

[34] For a study of shot noise in one-dımensional conductors, see V I Melnıkov, Phys Lett A 198 (1995) 459

[35] R C Liu, P Eastman and Y Yamamoto, preprint

[36] ON Do1okhov, Pis'ma Zh Eksp Teo1 Fiz 36 (1982) 259 [JETP Lett 36 (1982) 318], P A Mello, P Pcreyra and N Kumar, Ann Phys (N Y) 181 (1988) 290

[37] Y P Li, A Zaslavsky, D C Tsur, M Santos and M Shayegan, Phys Rev B 41 (1990) 8388

[38] H C Liu, J L1, G C Aers, C R Leavens, M Buchanan and Z R Wasılewsk1, Phys Rev B 51 (1995) 5116

[39] S Horshfield, J H Davies, P Hyldgaard, C J Stanton, and J W Wılkıns, Phys Rev B 47 (1992) 1967, A Levy Ycyatr, F Flores, and EV Anda, ibid 47 (1993) $10543, \mathrm{~K}-\mathrm{M}$ Hung and G Y Wu, rbid 48 (1993) 14687, U Hanke, Yu M Galpcim, K A Chao, and N Zou, rbıd 48 (1993) 17209 , U Hanke, Yu M Galperin and K A Chao, ıbid 50 (1994) 1595

[40] J A Melsen and C W J Beenakkeı, Physica B 203 (1994) 219

[41] C W I Beenakkeı, B Rejaeı and J A Melsen, Phys Rev Lett 72 (1994) 2470

[42] K E Nagacv, Phys Rev B 52 (1995) 4740

[43] V I Kozub and A M Rudin, Phys Rev B 52 (1995) 7853

[44] B L Altshuler, A G Aronov and DE Khmelnitsk11, J Phys C 15 (1982) 7367, A Stenn, Y Aharonov and Y Imry, Phys Rev B 41 (1990) 3436

[45] M Butt1ket, Phys Rev B 38 (1988) 9375

[46] M Buttıker, in Noisc in Physical Systems and $1 / f$ Fluctuations, eds V Barcik1s and R Katilius (World Scient1fic, Singapore, 1995)

[47] J H Davies, J Carlos Egues and J W Wılkıns, Phys Rev B 52 (1995) 11259 , see also J Caılos Egues, S Hershfield and JW Wilkins, bid 49 (1994) 13517

[48] See, e g, H van Houtcn, L W Molenkamp, C W J Beenakker and C T Foxon, Semıcond Scı Technol 7 (1992) B 215

[49] J M Martınıs and MH Devoret, unpubished

[50] A Steinbach, JM Maıtınıs and M H Devorct, Bull Am Phys Soc 40 (1995) 400, Phys Rev Lett 76 (1996) 3806

[51] R Landaue, Phys Rev B 47 (1993) 16427

[52] R C Liu and Y Yamamoto, Phys Rev B 49 (1994) 10520, 50 (1994) 17411

[53] A N Kolotkov, DV Averin, KK Likharev and SA Vasenko, in Single-Election Tunneling and Mesoscopic Devices, eds H Koch and H Lubbig (Spingeı, Beılın, 1992), A N Korotkov, Phys Rev B 49 (1994) 10381

[54] II Burk, M JM de Jong and C Schonenberger, Phys Rev Lett 75 (1995) 1610

[55] M Buttıker, in Noise in Physical Systems and 1/f Fluctuations, eds P II Handel and A L Chung (AIP, New York, 1993) 